\title{
A Color-to-Spin Domino Schensted Algorithm
}

\author{
Mark Shimozono * \\ Department of Mathematics \\ Virginia Tech \\ Blacksburg, VA 24061-0123 \\ mshimo@math.vt.edu
}

\author{
Dennis E. White \\ School of Mathematics \\ University of Minnesota \\ 127 Vincent Hall, 206 Church St SE \\ Minneapolis, MN 55455-0488 \\ white@math . umn.edu
}

Submitted: February 11, 2000; Accepted: May 29, 2001. MR Subject Classifications: Primary: 05E10; Secondary: 05E05.

\begin{abstract}
We describe the domino Schensted algorithm of Barbasch, Vogan, Garfinkle and van Leeuwen. We place this algorithm in the context of Haiman's mixed and leftright insertion algorithms and extend it to colored words. It follows easily from this description that total color of a colored word maps to the sum of the spins of a pair of 2-ribbon tableaux. Various other properties of this algorithm are described, including an alternative version of the Littlewood-Richardson bijection which yields the $q$-Littlewood-Richardson coefficients of Carré and Leclerc. The case where the ribbon tableau decomposes into a pair of rectangles is worked out in detail. This case is central in recent work [29] on the number of even and odd linear extensions of a product of two chains.
\end{abstract}

\section{Introduction}

In a 1982 paper Barbasch and Vogan [1] describe an insertion algorithm which identifies hyperoctahedral permutations (or "colored permutations") with domino tableaux. They define this insertion using left-right insertion of a word and its negative, followed by a jeu de taquin that pairs up $i$ and $-i$.

Subsequently Garfinkle [7] defined this insertion directly, both through a bumping algorithm (similar to Schensted [20] insertion) and recursively in a manner similar to that used by Fomin [4].

Van Leeuwen [27] also describes this algorithm by translating Garfinkle's recursive definition into Fomin's language of shapes. He provides the first proof that the Garfinkle

${ }^{*}$ Research supported by the NSF under grant number DMS-9800941 
algorithm is the same as the Barbasch-Vogan algorithm. He also defines insertion in the presence of a nonempty 2-core.

In this paper we give a self-contained treatment of this algorithm. Our interest in the algorithm is based on its color-to-spin property, which, to our knowledge, was not observed by these previous authors. That is, this algorithm identifies a hyperoctahedral permutation with a pair of domino tableaux so that the number of "bars" in the permutation (which we will call its total color) equals the sum of the spins of the tableaux.

We also place this algorithm in the context of Haiman's mixed insertion [8]. We generalize Haiman's insertions from colored permutations to biwords with colors on both the top and bottom lines. We describe a number of properties of this algorithm, including the fact that it can be used to give an alternative description of the domino LittlewoodRichardson bijection given by Carré and Leclerc [3].

Another domino insertion, described in [26], does not have this key color-to-spin property. Our investigations also led us to another color-to-spin algorithm, one which extends to $k$-ribbon tableaux, for any $k$. This algorithm is described in [22].

A consequence of this Schensted algorithm and its connection to $q$-Littlewood-Richardson coefficients is a correspondence between domino tableaux of rectangular shape, where one dimension is even, and standard Young tableaux of self-complementary shape. More generally, if the 2-quotient of the domino shape is a pair of rectangles, then the domino tableaux are in one-to-one correspondence with what we call semi-self-complementary standard tableaux.

The connection between domino tableaux of rectangular shape and semi-self-complementary standard tableaux follows easily from a result of Stanley [25] about the Littlewood-Richardson coefficients of pairs of (almost) equal rectangles. It also follows from recent work of Berenstein and Kirillov [2] on the connection between domino tableaux and self-evacuating tableaux under the Schützenberger involution. However, we proceed through the Barbasch-Vogan-Garfinkle algorithm so that the spin statistic is turned into a natural statistic on the standard tableau. We will call this statistic on standard tableaux of semi-self-complementary shape "twist." This spin-to-twist property is central to the proof that products of chains have their linear extensions sign-balanced if and only if the chain lengths are equal mod 2 [29].

Section 2 outlines the basic facts about partitions, words and tableaux which will be used throughout the paper. Haiman's insertion algorithms and their generalization to doubly colored biwords are described in Section 3. Domino tableaux, ribbon tableaux and the domino Schensted insertion are described in Section 4. The relationship to Haiman's insertion algorithms is also given here. The generalization to biwords and the connection to the $q$-analogues of the Littlewood-Richardson coefficients of Carré and Leclerc are given in Section 5. Finally, the special case of when the 2-quotient is a pair of rectangles is completely worked out in Section 6 . 


\section{Words and Tableaux}

In this section we will give the basic definitions and theorems for the combinatorial structures that arise in subsequent sections. The body of literature on this material is extensive. Our treatment follows Fulton [6], to which we refer the reader for the full statement and proof of many of the results below. Other sources are Sagan [19] (whose treatment is restricted to permutations), Macdonald [14] (whose emphasis is on symmetric functions) or Stanley [24] (which again emphasizes symmetric functions). Since many of these results have appeared in many places, and have been rediscovered many times, we have not been especially careful about attributions to original sources.

\subsection{Partitions, Words and Tableaux}

The sequence of integers $\lambda=\left(\lambda_{1} \geq \lambda_{2} \geq \cdots \geq \lambda_{t} \geq 0\right)$ is called a partition. The number of parts is the number of non-zero values. If $N=\sum_{i} \lambda_{i}$ then we say $\lambda$ partitions $N$ and we write $|\lambda|=N$ and $\lambda \vdash N$. Another notation for partitions is an exponential form to denote the parts and their multiplicities. For example, the partition $(4,4,3,1,1,1,1,1)$ is written $1^{5} 34^{2}$.

Yet another way of describing a partition is with a Ferrers diagram. A Ferrers diagram is an array of squares, left-justified, with $\lambda_{j}$ squares (or cells) in row $j$. For example, the Ferrers diagram for the partition $(4,4,3,1)$ is

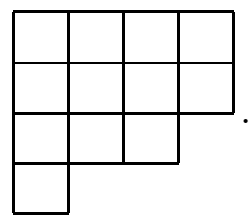

This pictorial description leads us to call partitions shapes.

If $\lambda$ is a shape and $\mu$ is a shape whose Ferrers diagram is contained in the Ferrers diagram of $\lambda$, then the skew shape $\lambda / \mu$ is the set of cells obtained by deleting the cells of $\mu$ from $\lambda$. For example, here is the skew shape $(6,6,4,2) /(5,2,1)$ :

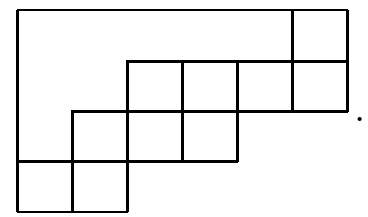

A word is a sequence of objects, not necessarily distinct, called letters. The letters have an order, so we usually use numbers for the letters. For example, 211334 is a word.

If the cells of a Ferrers diagram $\lambda$ are replaced by letters, the result is called a tableau of shape $\lambda$. A semistandard tableau is a tableau where the letters weakly increase across each row and strictly increase down each column. If $T$ is a tableau, then $\operatorname{sh}(T)$ is the shape of $T$. We let $S S_{\lambda}$ denote all the semistandard tableaux of shape $\lambda \vdash N$. Since we usually want this set to be finite, we restrict the set of letters to $\{1,2, \ldots, M\}$, where $M>|\lambda|$. 
The content of a word or tableau is a specification of the multiplicities of each letter. Thus, the word 211334 has content $(2,1,2,1)$, because there are two 1's, one 2, two 3's and one 4 . The content of the tableau

$$
T=\begin{array}{llll|}
1 & 1 & 2 & 2 \\
2 & 2 & 3 & \\
3 & & &
\end{array}
$$

is $(2,4,2)$, because $T$ has two 1's, four 2's, and two 3's.

A word or tableau is standard or uses a standard alphabet if no letter is used more than once. Standard words are also called permutations.

There are several ways to "read" the letters of a (skew) tableau which are compatible with the plactic monoid of the next subsection. We choose "column reading": read the letters from bottom to top, left to right. That is, first write down the letters in the leftmost column from bottom to top, then write down the letters in the next-to-leftmost column from bottom to top, etc. Let $w(T)$ denote this word.

(Although this is not the usual definition of the word of a tableau, it is compatible with the definition of the word of a ribbon tableau in Section 4. The usual definition is the "column reading" word, which is also compatible with the plactic monoid.)

For example, if

$$
T=\begin{array}{llll}
1 & 1 & 2 & 3 \\
2 & 3 & 3 & \\
4 & 4 &
\end{array},
$$

then $w(T)=421431323$.

\subsection{The Plactic Monoid}

We now describe an equivalence relation on words. The word $\mathbf{w}$ is type 1 equivalent to the word $\mathbf{v}$ if $\mathbf{w}$ contains the subsequence $b a c$, with $a<b \leq c$, and $\mathbf{v}$ is the same as $\mathbf{w}$, except that it contains the subsequence $b c a$. The word $\mathbf{w}$ is type 2 equivalent to the word $\mathbf{v}$ if $\mathbf{w}$ contains the subsequence $a c b$, with $a \leq b<c$, and $\mathbf{v}$ is the same as $\mathbf{w}$, except that it contains the subsequence $c a b$. Then $\mathbf{w}$ and $\mathbf{v}$ are Knuth equivalent, or simply equivalent, written $\mathbf{w} \stackrel{\mathrm{s}}{\sim} \mathbf{v}$, if $\mathbf{w}$ can be obtained from $\mathbf{v}$ by a sequence of type 1 and type 2 equivalences. Knuth equivalence was introduced by Knuth [10] to describe when two words had the same insertion tableau under the Schensted correspondence, a fact we shall arrive at shortly.

Under the operation juxtaposition, denoted by $\cdot$, the set of words form a free associative monoid. The quotient of this monoid under Knuth equivalence is called the plactic monoid.

The elements of the plactic monoid may be regarded as semistandard tableaux. This description is due to Lascoux and Schützenberger [12].

Theorem 1. For any word $\mathbf{w}$ there is a unique semistandard tableau $T$, with the same content, such that $w(T) \stackrel{\text { s }}{\sim} \mathbf{w}$. 
Theorem 1 motivates defining an associative multiplication on semistandard tableaux, $R=S \cdot T$, so that $w(R) \stackrel{\mathcal{S}}{\sim} w(S) \cdot w(T)$. This multiplication may be described directly using Schensted row or column insertion.

\subsection{Row and Column Insertion}

Schensted row insertion can be defined as follows If $x$ is a letter and $T$ a semistandard tableau, we construct the semistandard tableau $(T \stackrel{\mathrm{s}}{\leftarrow} x)$ through a series of "bumps." That is, $x$ is placed into the first row, replacing, or "bumping," the smallest letter $y$ strictly greater than $x$. Then $y$ is placed in the second row, bumping the smallest letter strictly greater than $y$ into the third row, and so on. The process stops when the letter entering a given row is $\geq$ all the letters in the row, in which case it is placed at the end of the row. A precise description of this algorithm may be found in [6], [19], and many other places.

A column dual of this algorithm, called Schensted column insertion, replaces rows with columns, and switches strict and non-strict inequalities. We write $(x \stackrel{s}{\rightarrow} T)$ to denote the resulting semistandard tableau.

Proposition 2. Let $x$ represent both the letter $x$ and the tableau consisting of a single cell containing $x$ and let $T$ be a semistandard tableau. Then $(T \stackrel{\mathrm{s}}{\leftarrow} x)=T \cdot x$ and $(x \stackrel{\mathrm{s}}{\rightarrow} T)=x \cdot T$.

Corollary 3. Row and column insertion commute, that is, for letters $x$ and $y$ and semistandard tableau $T,(x \stackrel{\mathrm{s}}{\rightarrow}(T \stackrel{\mathrm{s}}{\leftarrow} y))=((x \stackrel{\mathrm{s}}{\rightarrow} T) \stackrel{\mathrm{s}}{\leftarrow} y)$.

Proof. Both tableaux are $x \cdot T \cdot y$ and $\cdot$ is associative.

If $T$ is semistandard and $x$ and $y$ are two letters, let $T^{\prime}=(T \stackrel{\mathrm{s}}{\leftarrow} x)$ and $T^{\prime \prime}=\left(T^{\prime} \stackrel{\mathrm{s}}{\leftarrow} y\right)$. The shape of $T^{\prime}$ will differ from the shape of $T$ by a single cell $c$, while the shape of $T^{\prime \prime}$ will differ from the shape of $T^{\prime}$ by a single cell $c^{\prime}$.

Proposition 4. If $x \leq y$, then $c^{\prime}$ lies in a column strictly to the right of $c$ and in a row weakly above $c$. If $x>y$, then $c^{\prime}$ lies in a column weakly to the left of $c$ and in a row strictly below $c$.

Now define the insertion tableau for a word $\mathbf{w}=w_{1} w_{2} \ldots w_{n}$,

$$
P_{s}(\mathbf{w})=\left(\left(\ldots\left(\left(\emptyset \stackrel{\mathrm{s}}{\leftarrow} w_{1}\right) \stackrel{\mathrm{s}}{\leftarrow} w_{2}\right) \ldots\right) \stackrel{\mathrm{s}}{\leftarrow} w_{n}\right) .
$$

Corollary 5. The insertion tableau $P_{s}(\mathbf{w})$ is the unique semistandard tableau $T$ for $\mathbf{w}$ given by Theorem 1. Also,

$$
P_{s}(\mathbf{w})=\left(w_{1} \stackrel{\mathrm{s}}{\rightarrow}\left(\ldots\left(w_{n-1} \stackrel{\mathrm{s}}{\rightarrow}\left(w_{n} \stackrel{\mathrm{s}}{\rightarrow} \emptyset\right)\right) \ldots\right)\right) .
$$


We say the word $\mathbf{w}$ is a reverse lattice word if, at every point in the word when reading the word from right to left, the number of 1's is greater than or equal to the number of 2's, the number of 2's is greater than or equal to the number of 3's, etc. Also, we say a semistandard (skew) tableau $T$ is Yamanouchi if $w(T)$ is a reverse lattice word. It is easy to see that non-skew semistandard $T$ is Yamanouchi if and only if $T$ consists of 1 's in the first row, 2's in the second row, etc.

Proposition 6. The word $\mathbf{w}$ is a reverse lattice word if and only if $P_{s}(\mathbf{w})$ is Yamanouchi.

A second construction, called jeu de taquin, and defined by Schützenberger [23], can also be used to describe plactic multiplication. Since it is not necessary for our exposition, we omit its description.

\subsection{Biwords and the Schensted Correspondence}

A biletter ${ }_{j}^{i}$ is a $2 \times 1$ array of letters. The two letters are referred to as the top letter and the bottom letter. A biword is a sequence of biletters, with biletters sorted lexicographically. That is, the biletter ${ }_{j}^{i}$ precedes the biletter ${ }_{l}^{k}$ if one of the following two conditions holds:

i. $i<k$

ii. $i=k$ and $j<l$.

For example,

$$
\mathbf{w}=\left(\begin{array}{llllllll}
1 & 1 & 1 & 1 & 2 & 3 & 3 & 3 \\
1 & 1 & 2 & 3 & 3 & 2 & 2 & 3
\end{array}\right)
$$

is a biword. The upper word is the top row, the lower word the bottom row. We may speak of the content of the upper word and the content of the lower word.

If we turn all the biletters of a biword $\mathbf{w}$ upside down and sort according to the biword rules, we have described a new biword, which we call the inverse, ${ }^{\text {inv }}$. In the above example,

$$
\mathbf{w}^{\text {inv }}=\left(\begin{array}{llllllll}
1 & 1 & 2 & 2 & 2 & 3 & 3 & 3 \\
1 & 1 & 1 & 3 & 3 & 1 & 2 & 3
\end{array}\right) .
$$

The operator inv is an involution. If the lower word of $\mathbf{w}$ is a permutation of $\{1,2, \ldots, n\}$ and the upper word is $1,2, \ldots, n$, then the lower word of $\mathbf{w}^{\text {inv }}$ is the usual algebraic inverse of the lower word of $\mathbf{w}$.

If $\mathbf{w}$ is a biword, define $P_{s}(\mathbf{w})$ to be $P_{s}$ applied to the lower word of $\mathbf{w}$. Suppose ${ }_{j}^{i}$ is a biletter in $\mathbf{w}$. When $j$ is inserted in the construction of $P_{s}(\mathbf{w})$, a new shape is created, one cell larger than the previous shape. This shape difference is recorded in another tableau by placing $i$ in the new cell. This second tableau is called the recording tableau. The recording tableau is denoted by $Q_{s}(\mathbf{w})$. The content of $Q_{s}(\mathbf{w})$ will be the content of the upper word, while the content of $P_{s}(\mathbf{w})$ will be the content of the lower word. A 
consequence of Proposition 4 and the definition of biwords is that $Q_{s}(\mathbf{w})$ is semistandard. We have therefore identified a biword $\mathbf{w}$ with a pair of semistandard tableaux of the same shape.

An early version of this correspondence for words appeared in the work of Robinson [17]. It was rediscovered by Schensted [20], who described it on permutations. Knuth [10] then extended it to general biwords. We will call it the RSK-correspondence.

Theorem 7. The RSK-correspondence is a bijection between biwords $\mathbf{w}$ and pairs of semistandard tableaux, $P_{s}(\mathbf{w})$ and $Q_{s}(\mathbf{w})$. The content of the upper word of $\mathbf{w}$ is the same as the content of $Q_{s}(\mathbf{w})$ and the content of the lower word of $\mathbf{w}$ is the same as the content of $P_{s}(\mathbf{w})$. The shape of $P_{s}(\mathbf{w})$ equals the shape of $Q_{s}(\mathbf{w})$.

One of the most important properties of the RSK-correspondence is a symmetry property.

Theorem 8. We have

$$
P_{s}\left(\mathbf{w}^{\mathrm{inv}}\right)=Q_{s}(\mathbf{w})
$$

and

$$
Q_{s}\left(\mathbf{w}^{\text {inv }}\right)=P_{s}(\mathbf{w})
$$

\subsection{Standardization}

Let $\mathbf{w}$ be a word. Write $\mathbf{w}^{\text {st }}$ to denote the standardization of $\mathbf{w}$. That is, convert the letters of $\mathbf{w}$ to a standard alphabet, first converting all the smallest letters, from left to right, then the next smallest, etc.

If $\mathbf{w}$ is a biword, standardization is computed by converting both the upper word and the lower word to standard alphabets. Again, we use the notation $\mathbf{w}^{\mathrm{st}}$.

If $T$ is a semistandard (skew) tableau, then $T^{\text {st }}$ is the tableau obtained by converting the letters to a standard alphabet, where all the smallest letters are converted first, from left to right.

Standardization is compatible with all the constructions described above.

\section{Proposition 9.}

$$
\begin{gathered}
\text { If } \mathbf{w} \stackrel{\mathrm{s}}{\sim} \mathbf{v} \text { then } \mathbf{w}^{\mathrm{st}} \stackrel{\mathrm{s}}{\sim} \mathbf{w}^{\mathrm{st}} \\
w\left(T^{\mathrm{st}}\right) \stackrel{\mathrm{s}}{\sim} w(T)^{\mathrm{st}} \\
J\left(T^{\mathrm{st}}\right)=J(T)^{\mathrm{st}} \\
P_{s}\left(\mathbf{w}^{\mathrm{st}}\right)=P_{s}(\mathbf{w})^{\mathrm{st}} \\
Q_{s}\left(\mathbf{w}^{\mathrm{st}}\right)=Q_{s}(\mathbf{w})^{\mathrm{st}}
\end{gathered}
$$




\subsection{Schur Functions}

If $T$ is a semistandard tableau with content $\left(c_{1}, \ldots, c_{N}\right)$, and $\mathbf{x}=\left\{x_{1}, x_{2}, \ldots\right\}$ is a set of indeterminates, then define

$$
\mathbf{x}^{T}=x_{1}^{c_{1}} x_{2}^{c_{2}} \ldots x_{N}^{c_{N}}
$$

The monomial $\mathbf{x}^{T}$ is called the weight of $T$. For instance, for

$$
T=\begin{array}{llll}
1 & 1 & 2 & 2 \\
2 & 2 & 3 & \\
3 & & &
\end{array},
$$

we have $\mathbf{x}^{T}=x_{1}^{2} x_{2}^{4} x_{3}^{2}$.

If we sum these weights over all the semistandard tableaux of shape $\lambda \vdash N$, we obtain the Schur function. That is,

$$
s_{\lambda}(\mathbf{x})=\sum_{T \in S S_{\lambda}} \mathbf{x}^{T} .
$$

The Schur functions are symmetric functions and, in fact, the set $\left\{s_{\lambda}\right\}_{\lambda \vdash N}$ forms a basis for the symmetric functions homogeneous of degree $N$ (see [14]). In a similar fashion, we can define skew Schur functions.

When two Schur functions are multiplied, the resulting symmetric function can be expanded in the Schur function basis. The coefficients are called the Littlewood-Richardson coefficients. That is,

$$
s_{\mu}(\mathbf{x}) s_{\nu}(\mathbf{x})=\sum_{\lambda} c_{\mu, \nu}^{\lambda} s_{\lambda}(\mathbf{x}) .
$$

The mapping $T \longrightarrow \mathbf{x}^{T}$ defines a ring homomorphism from the group ring of the plactic monoid to the polynomial ring.

Corollary 10. If $T$ is semistandard of shape $\lambda$,

$$
c_{\mu, \nu}^{\lambda}=\#\left\{(U, V): U \in S S_{\mu}, V \in S S_{\nu} \text { and } U \cdot V=T\right\} .
$$

\section{Haiman's Insertion Algorithms}

In this section we describe Haiman's insertion algorithms. We first define colored words, biwords and tableaux. We also introduce doubly colored biwords. Then we define Haiman's mixed and left-right insertions, and give some of their properties. We conclude this section with a generalization of Haiman's insertion algorithms, which we call doubly mixed insertion, and we prove some if its properties.

\subsection{Colored Words}

A fundamental object considered in this paper is a colored word. A colored word is a word with bars over some of the letters. A letter in such a word is called a colored letter. 
A colored letter may be barred or unbarred. We adopt the following convention for the order of letters in a colored word:

$$
\overline{1}<1<\overline{2}<2<\cdots<\bar{n}<n .
$$

An example of a colored word is

$$
\mathbf{w}=\overline{4} 2 \overline{2} \overline{1} \overline{4} 32 .
$$

A special case of a colored word is a colored permutation. A colored permutation is a colored word in which each letter (either barred or unbarred) is used no more than once.

If $\mathbf{w}$ is a colored word, we write $t c(\mathbf{w})$ to denote the total color of the word, that is, the number of barred letters in the word. In the above example, $t c(\mathbf{w})=4$.

If $\mathbf{w}$ is a colored word (resp. letter), we write $\mathbf{w}^{\text {neg }}$ to denote the word (resp. letter) obtain by converting the bars to negative signs.

More generally, a colored biword is a two row array with some of the letters on the lower word barred and such that if the bars are replaced by negative signs, the result is a biword.

For example,

$$
\mathbf{w}=\left(\begin{array}{lllllll}
1 & 1 & 1 & 2 & 2 & 2 & 2 \\
\overline{2} & \overline{1} & 2 & \overline{3} & \overline{3} & \overline{1} & 2
\end{array}\right)
$$

is a colored biword.

We extend the definition of neg to colored biwords in the obvious way. For example, if $\mathbf{w}$ is as given above, then

$$
\mathbf{w}^{\text {neg }}=\left(\begin{array}{ccccccc}
1 & 1 & 1 & 2 & 2 & 2 & 2 \\
-2 & -1 & 2 & -3 & -3 & -1 & 2
\end{array}\right) .
$$

The definition of colored biword guarantees that $\mathbf{w}^{\text {neg }}$ will be a biword.

Even more generally, a doubly colored biword $\mathbf{w}$ is a two row array with some of the letters in each row barred, and with the biletters sorted according to the following rule. The biletter ${ }_{j}^{i}$ precedes the biletter ${ }_{l}^{k}$ if one of the following three conditions holds:

i. $i<k$

ii. $i=k$, both are unbarred, and $j^{\text {neg }}<l^{\text {neg }}$

iii. $i=k$, both are barred, and $l^{\text {neg }}<j^{\text {neg }}$

An example of a doubly colored biword is

$$
\mathbf{w}=\left(\begin{array}{lllllllll}
\overline{1} & \overline{1} & \overline{1} & \overline{1} & 1 & \overline{2} & \overline{2} & 2 & 2 \\
2 & 1 & \overline{1} & \overline{3} & \overline{2} & 2 & \overline{1} & 1 & 2
\end{array}\right)
$$

Now extend the definition of neg to doubly colored biwords by converting the bars in the lower word to negatives. The resulting word is a doubly colored biword, with the bars 
only appearing on the upper word. Also note that neg is invertible: simply replace the negatives with bars.

In the example above,

$$
\mathbf{w}^{\text {neg }}=\left(\begin{array}{ccccccccc}
\overline{1} & \overline{1} & \overline{1} & \overline{1} & 1 & \overline{2} & \overline{2} & 2 & 2 \\
2 & 1 & -1 & -3 & -2 & 2 & -1 & 1 & 2
\end{array}\right) .
$$

We also define the "inverse" of a doubly colored biword. Let $\mathbf{w}^{\text {inv }}$ be the doubly colored biword obtained by writing the lower word of $\mathbf{w}$ as the upper word, the upper word of $\mathbf{w}$ as the lower word, and sorting the biletters according to the rules for doubly colored biwords. Continuing the previous example,

$$
\mathbf{w}^{\mathrm{inv}}=\left(\begin{array}{lllllllll}
\overline{1} & \overline{1} & 1 & 1 & \overline{2} & 2 & 2 & 2 & \overline{3} \\
\overline{1} & \overline{2} & \overline{1} & 2 & 1 & \overline{2} & \overline{1} & 2 & \overline{1}
\end{array}\right) .
$$

The operator inv is an involution on doubly colored biwords. Also the operator inv neg inv effectively negates the barred letters on the upper word, then sorts according to the colored biword rules, thus producing a colored biword. In the above example,

$$
\mathbf{w}^{\text {inv neg inv }}=\left(\begin{array}{ccccccccc}
-2 & -2 & -1 & -1 & -1 & -1 & 1 & 2 & 2 \\
\overline{1} & 2 & \overline{3} & \overline{1} & 1 & 2 & \overline{2} & 1 & 2
\end{array}\right) .
$$

Another operation defined on doubly colored biwords is "evacuation." Define $\mathbf{w}^{\mathrm{ev}}$ to be the doubly colored biword obtained by removing all the biletters whose lower letter is barred. In the above example,

$$
\mathbf{w}^{\mathrm{ev}}=\left(\begin{array}{lllll}
\overline{1} & \overline{1} & \overline{2} & 2 & 2 \\
2 & 1 & 2 & 1 & 2
\end{array}\right)
$$

An easy fact is the following remark.

Proposition 11. The operations ev and neg both commute with inv neg inv.

It is sometimes necessary to standardize a doubly colored biword. This is accomplished by describing a partial standardization, of the upper word only. Let $\mathbf{w}^{\overline{\mathrm{st}}}$ describe replacing the upper word of $\mathbf{w}$ with a standard alphabet, with the positions of the bars remaining. In the above example,

$$
\mathbf{w}^{\overline{\mathrm{st}}}=\left(\begin{array}{lllllllll}
\overline{1} & \overline{2} & \overline{3} & \overline{4} & 5 & \overline{6} & \overline{7} & 8 & 9 \\
2 & 1 & \overline{1} & \overline{3} & \overline{2} & 2 & \overline{1} & 1 & 2
\end{array}\right) .
$$

Now we can standardize the lower word by switching the lower and upper words using inv, doing a partial standardization, $\overline{\text { st}}$, then switching back. Therefore, define

$$
\mathbf{w}^{\text {st }}=\mathbf{w}^{\overline{\text { st inv }} \overline{\text { st inv }}} \text {. }
$$

In the above example,

$$
\mathbf{w}^{\text {st }}=\left(\begin{array}{lllllllll}
\overline{1} & \overline{2} & \overline{3} & \overline{4} & 5 & \overline{6} & \overline{7} & 8 & 9 \\
7 & 3 & \overline{1} & \overline{9} & \overline{5} & 6 & \overline{2} & 4 & 8
\end{array}\right)
$$

THE ELECTRONiC JOURNAL OF COMBINATORICS 8 (2001), \#R21 
Proposition 12. The operator st commutes with inv. It also commutes with neg and ev on doubly colored biwords with proper choice of standardizing alphabet. Finally, st has the alternative definition:

$$
\mathbf{w}^{\mathrm{st}}=\mathbf{w}^{\text {inv } \overline{\mathrm{st}} \operatorname{inv} \overline{\mathrm{st}}} .
$$

\subsection{Colored Tableaux}

A colored tableau is a tableau with colored letters. We define the operators neg, ev and st on semistandard colored tableaux in terms of Haiman's conversion operators.

Haiman [8] describes a conversion process in which one letter in a semistandard tableau is "replaced" by another. This process proceeds as follows. Let $x$ be the letter in cell $c$ in a semistandard tableau $T$ and let $y$ be another letter. Replace $x$ with $y$ in the cell $c$. The resulting tableau may not be semistandard. Therefore, swap the $y$ in cell $c$ with one of its neighbors (above or to the left, if $y$ is smaller than $x$; below or to the right, if $y$ is larger than $x$ ). Now $y$ is in a new cell, $c^{\prime}$. Again, the tableau may not be semistandard. Therefore, repeat this swapping until the tableau is restored to semistandard. We will say that the value $x$ was converted to $y$. (Conversion may also be described in terms of jeu de taquin slides.)

We define neg on semistandard colored tableau as a sequence of conversions. Suppose $T$ is a semistandard colored tableau. Let $T^{\text {neg }}$ be the semistandard tableau obtained by successively converting the barred letters $x$ in $T$ to their corresponding negatives, $x^{\text {neg }}$. The barred letters are converted from smallest to largest. Repeated letters are converted from left to right in the tableau. For example, if

$$
T=\begin{array}{llllll|}
\hline \overline{1} & \overline{1} & \overline{1} & 1 & 1 & 2 \\
1 & \overline{2} & 2 & \overline{3} & & \\
\overline{2} & 2 & \overline{3} & & & \\
\overline{3} & 3 & 3 & & & \\
& & & &
\end{array}
$$

then

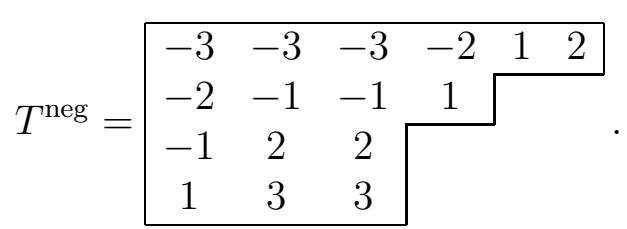

Note that neg is invertible.

The operator ev is defined in a similar fashion. Let $T^{\mathrm{ev}}$ be the semistandard tableau obtained by successively converting the barred letters to $+\infty$ (larger than any letter in the tableau), then erasing the $+\infty$. The barred letters are converted from largest to smallest. Repeated letters are converted from right to left. In the above example,

$$
T^{\mathrm{ev}}=\begin{array}{llll}
1 & 1 & 1 & 2 \\
2 & 2 & & \\
3 & 3 & &
\end{array} .
$$

THE ELECTRONIC JOURNAL OF COMBINATORICS 8 (2001), \#R21 
Finally, let $T^{\text {st }}$ be the usual standardization of $T$, where a letter will retain its "color" after being replaced by the standardizing alphabet. In the above example,

$$
T^{\text {st }}=\begin{array}{cccccc}
\overline{1} & \overline{2} & \overline{3} & 5 & 6 & 11 \\
4 & \overline{8} & 10 & \overline{14} & & \\
\overline{7} & 9 & \overline{13} & & & \\
\overline{12} & 15 & 16 & & & \\
\end{array}
$$

Proposition 13. With appropriate choice of standardizing alphabet, ev and neg commute with st on colored tableaux.

\subsection{Mixed and Left-Right Insertion}

Most of the material in this subsection is due to Haiman [8]. Haiman described his insertion algorithms for colored permutations with no repeated letters, but noted that extensions to words were straightforward. We will use these extensions to words in this subsection.

Also, Haiman described two kinds of insertion, mixed and left-right, but noted that a more general combination of the two was possible. We will describe this more general insertion, which we will call "doubly mixed insertion," in the next subsection.

First, however, we describe Haiman's mixed and left-right insertions. Suppose $T$ is a semistandard colored tableau and suppose $x$ is a colored letter. If $x$ is barred, it is inserted into the first column. If it is unbarred, it is inserted into the first row. Subsequent letters are bumped into the next column or row according to whether they are barred or unbarred.

The resulting semistandard colored tableau $(T \stackrel{\mathrm{m}}{\leftarrow} x)$ will include the same colored letters as $T$, with the addition of the colored letter $x$. For example, if

$$
T=\begin{array}{lll}
1 & \overline{2} & 2 \\
\overline{2} & \overline{3} & \\
2 & &
\end{array},
$$

and we wish to construct $(T \stackrel{m}{\leftarrow} \overline{1})$, then

$\overline{1}$ bumps 1 in location $(1,1)$

1 bumps $\overline{2}$ in location $(2,1)$

$\overline{2}$ bumps $\overline{2}$ in location $(1,2)$

$\overline{2}$ bumps 2 in location $(1,3)$

2 bumps $\overline{3}$ in location $(2,2)$ and

$\overline{3}$ is placed in location $(2,3)$. 
Therefore,

$$
(T \stackrel{m}{\leftarrow} \overline{1})=\left[\begin{array}{lll}
\overline{1} & \overline{2} & \overline{2} \\
1 & 2 & \overline{3} \\
2 &
\end{array} .\right.
$$

Now for colored word $\mathbf{w}=w_{1} w_{2} \ldots w_{n}$, define

$$
P_{m}(\mathbf{w})=\left(\left(\ldots\left(\left(\emptyset \stackrel{\mathrm{m}}{\leftarrow} w_{1}\right) \stackrel{\mathrm{m}}{\leftarrow} w_{2}\right) \ldots\right) \stackrel{\mathrm{m}}{\leftarrow} w_{n}\right) .
$$

For example, if $\mathbf{w}=2 \overline{2} 1 \overline{3} \overline{2} 2 \overline{1}$, then

$$
P_{m}(\mathbf{w})=\begin{array}{lll}
\overline{1} & \overline{2} & \overline{2} \\
1 & 2 & \overline{3} \\
2 &
\end{array}
$$

This insertion process is called mixed insertion. We clearly have that $P_{m}(\mathbf{w})$ is a semistandard colored tableau. We write $Q_{m}(\mathbf{w})$ to denote the corresponding recording tableau, using a standard alphabet. If $\mathbf{w}$ is a colored biword, then $P_{m}(\mathbf{w})$ is the mixed insertion tableau of the lower word, while $Q_{m}(\mathbf{w})$ is the recording tableau for this mixed insertion, using the upper word as the recording alphabet.

For example, if

$$
\mathbf{w}=\left(\begin{array}{lllllll}
1 & 1 & 1 & 2 & 2 & 3 & 3 \\
\overline{3} & \overline{1} & 2 & \overline{2} & 1 & \overline{2} & 2
\end{array}\right)
$$

then

$$
P_{m}(\mathbf{w})=\begin{array}{llll}
\overline{1} & 1 & 2 & \overline{3} \\
\overline{2} & \overline{2} & & \\
2 & &
\end{array} \quad \text { and } \quad Q_{m}(\mathbf{w})=\begin{array}{llll}
1 & 1 & 1 & 3 \\
2 & 2 & & \\
3 & & &
\end{array} .
$$

An easy consequence of Haiman's Theorem 3.12 connects mixed insertion with ordinary RSK-insertion.

Proposition 14. If $\mathbf{w}$ is a colored biword, then

$$
P_{m}(\mathbf{w})^{\mathrm{neg}}=P_{s}\left(\mathbf{w}^{\mathrm{neg}}\right)
$$

and

$$
Q_{m}(\mathbf{w})=Q_{s}\left(\mathbf{w}^{\text {neg }}\right) .
$$

Note that $Q_{m}(\mathbf{w})$ is semistandard, from the definition of colored biwords, Proposition 4, and Proposition 14. Also, mixed insertion commutes with standardization of colored biwords.

Proposition 15. The operator st commutes with $P_{m}$ and $Q_{m}$. 
Proof. For $P_{m}$, since st commutes with neg on colored words and colored tableaux, the result follows from Proposition 14, Proposition 9 and the invertibility of neg. The proof for $Q_{m}$ is similar.

If two colored words $\mathbf{w}$ and $\mathbf{v}$ have the same mixed insertion tableau, i.e., $P_{m}(\mathbf{w})=$ $P_{m}(\mathbf{v})$, then they are mixed equivalent and we write $\mathbf{w} \stackrel{\mathrm{m}}{\sim} \mathbf{v}$. Since the operator neg is invertible on tableaux, we have the following corollary to Proposition 14.

Corollary 16. Suppose $\mathbf{w}$ and $\mathbf{v}$ are colored words. Then $\mathbf{w} \stackrel{\mathrm{m}}{\sim} \mathbf{v}$ if and only if $\mathbf{w}^{\text {neg }} \stackrel{\mathrm{s}}{\sim}$ $\mathbf{v}^{\text {neg }}$.

The following is Haiman's Corollary 3.18.

Proposition 17. If $\mathbf{w}$ is a colored biword, then

$$
P_{m}(\mathbf{w})^{\mathrm{ev}}=P_{s}\left(\mathbf{w}^{\mathrm{ev}}\right) .
$$

Haiman's second insertion process is called left-right insertion. A doubly colored biword $\mathbf{w}$ is called upper colored if $\mathbf{w}^{\text {inv }}$ is a colored biword. That is, $\mathbf{w}$ has colors only on the upper word. Left-right insertion is defined on upper colored biwords. If $T$ is a semistandard tableau, define

$$
\left(T \stackrel{\ln }{\leftarrow} \begin{array}{l}
\bar{i} \\
x
\end{array}\right)=(x \stackrel{\mathrm{s}}{\longrightarrow} T)
$$

and

$$
(T \stackrel{\operatorname{lr}}{\leftarrow} \underset{x}{i})=(T \stackrel{\mathrm{s}}{\leftarrow} x)
$$

Let $P_{l r}(\mathbf{w})$ denote the insertion tableau and $Q_{l r}(\mathbf{w})$ the recording tableau. The colors are kept in the recording tableau, so that $Q_{l r}(\mathbf{w})$ is a colored semistandard tableau. For example, if

$$
\mathbf{w}=\left(\begin{array}{lllllll}
\overline{1} & \overline{1} & \overline{1} & 1 & \overline{2} & 2 & 2 \\
3 & 2 & 2 & 1 & 3 & 1 & 2
\end{array}\right)
$$

then

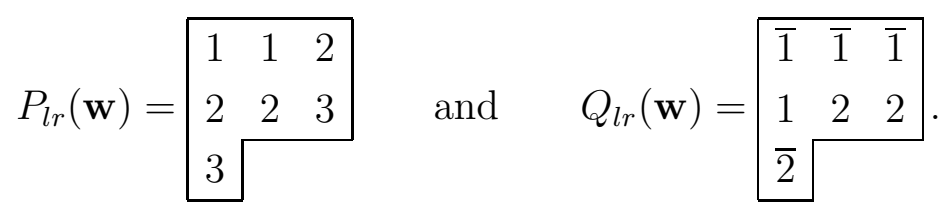

Haiman showed that mixed insertion and left-right insertion were, in effect, dual algorithms. The following proposition is Haiman's Theorem 4.3.

Proposition 18. If $\mathbf{w}$ is a colored biword, then

$$
P_{m}(\mathbf{w})=Q_{l r}\left(\mathbf{w}^{\mathrm{inv}}\right)
$$

and

$$
Q_{m}(\mathbf{w})=P_{l r}\left(\mathbf{w}^{\text {inv }}\right)
$$


Proposition 19. The tableau $Q_{l r}\left(\mathbf{w}^{\mathrm{inv}}\right)$ is semistandard. Also, st commutes with $P_{l r}$ and $Q_{l r}$.

Proof. That the tableau $Q_{l r}\left(\mathbf{w}^{\mathrm{inv}}\right)$ is semistandard follows from Proposition 18. That st commutes with $P_{l r}$ and $Q_{l r}$ follows from Proposition 12, Proposition 15 and Proposition 18.

\subsection{Doubly Mixed Insertion}

We now extend Haiman's results to doubly colored biwords. Haiman remarked that this extension could be done, but had no need for it. Since we will find this extension useful, we make Haiman's remarks precise.

Suppose $T$ is a colored semistandard tableau and ${ }_{x}^{i}$ is a doubly colored biletter. Then define

$$
\left(T \stackrel{\mathrm{m}^{*}}{\leftarrow} \underset{x}{i}\right)=\left\{\begin{array}{ll}
(T \stackrel{\mathrm{m}}{\leftarrow} x) & \text { if } i \text { is not barred } \\
(T \stackrel{\mathrm{dm}}{\leftarrow} x) & \text { if } i \text { is barred }
\end{array},\right.
$$

where $\stackrel{d m}{\leftarrow}$ is a "dual" mixed insertion in which the barred letters bump by rows and the unbarred letters bump by columns. As usual, define $P_{m^{*}}(\mathbf{w})$ and $Q_{m^{*}}(\mathbf{w})$ for a doubly colored biword w. In this case, both tableaux will be colored. For example, if

$$
T=\begin{array}{llll}
\overline{1} & 1 & \overline{2} & 3 \\
1 & 2 & 2 & \\
\overline{3} & & &
\end{array},
$$

then

$$
\left(T \stackrel{\mathrm{m}^{*}}{\leftarrow} \overline{\overline{3}} \overline{\overline{1}}\right)=\begin{array}{lllll}
\hline \overline{1} & \overline{1} & 1 & 2 & 3 \\
1 & \overline{2} & 2 & & \\
\overline{3} & & & &
\end{array} .
$$

If

$$
\mathbf{w}=\left(\begin{array}{lllllllll}
\overline{1} & \overline{1} & 1 & \overline{2} & 2 & 2 & \overline{3} & \overline{3} & \overline{3} \\
\overline{1} & \overline{2} & 1 & 2 & \overline{3} & 3 & 2 & 1 & \overline{1}
\end{array}\right)
$$

then

$$
P_{m^{*}}(\mathbf{w})=\begin{array}{lllll|}
\overline{1} & \overline{1} & 1 & 2 & 3 \\
1 & \overline{2} & 2 & & \\
\overline{3} & & & &
\end{array}
$$

and

$$
Q_{m^{*}}(\mathbf{w})=\begin{array}{lllll}
\overline{1} & \overline{1} & 1 & 2 & \overline{3} \\
\overline{2} & \overline{3} & \overline{3} & & \\
2 & & & &
\end{array} .
$$


Proposition 20. If $\mathbf{w}$ is a colored biword, then $P_{m^{*}}(\mathbf{w})=P_{m}(\mathbf{w})$ and $Q_{m^{*}}(\mathbf{w})=Q_{m}(\mathbf{w})$. Similarly, if $\mathbf{w}$ is an upper colored biword, then $P_{m^{*}}(\mathbf{w})=P_{l r}(\mathbf{w})$ and $Q_{m^{*}}(\mathbf{w})=Q_{l r}(\mathbf{w})$.

Proof. The first part is true since doubly mixed insertion and mixed insertion are the same on colored biwords. The second part is true since doubly mixed insertion and left-right insertion are the same on upper colored biwords.

Doubly mixed insertion can be realized as mixed insertion or left-right insertion.

Theorem 21. If $\mathbf{w}$ is a doubly colored biword, then

$$
\begin{gathered}
P_{m^{*}}(\mathbf{w})=P_{m}\left(\mathbf{w}^{\text {inv neg inv }}\right) \\
P_{m^{*}}(\mathbf{w})^{\text {neg }}=P_{l r}\left(\mathbf{w}^{\text {neg }}\right)=P_{m^{*}}\left(\mathbf{w}^{\text {neg }}\right) \\
P_{m^{*}}(\mathbf{w})=Q_{m^{*}}\left(\mathbf{w}^{\text {inv }}\right) \\
Q_{m^{*}}(\mathbf{w})=Q_{l r}\left(\mathbf{w}^{\text {neg }}\right) \\
Q_{m^{*}}(\mathbf{w})^{\text {neg }}=Q_{m}\left(\mathbf{w}^{\text {inv neg inv }}\right)=Q_{m^{*}}\left(\mathbf{w}^{\text {inv neg inv }}\right) \\
Q_{m^{*}}(\mathbf{w})=P_{m^{*}}\left(\mathbf{w}^{\text {inv }}\right)
\end{gathered}
$$

Proof. Equation (1) is a consequence of Haiman's Remark 8.5. The first identity in Equation (2) follows from Equation (1) since

$$
\begin{aligned}
P_{m}\left(\mathbf{w}^{\text {inv neg inv }}\right)^{\text {neg }} & =P_{s}\left(\mathbf{w}^{\text {inv neg inv neg }}\right) & & \text { by Proposition } 14 \\
& =P_{s}\left(\mathbf{w}^{\text {neg inv neg inv }}\right) & & \text { by Proposition } 11 \\
& =Q_{s}\left(\mathbf{w}^{\text {neg inv neg }}\right) & & \text { by Theorem } 8 \\
& =Q_{m}\left(\mathbf{w}^{\text {neg inv }}\right) & & \text { by Proposition } 14 \\
& =P_{l r}\left(\mathbf{w}^{\text {neg }}\right) & & \text { by Proposition } 18 .
\end{aligned}
$$

The second identity follows from Proposition 20.

From Equation (2), the shape change in $P_{m^{*}}(\mathbf{w})$ is the same as the shape change in $P_{l r}\left(\mathbf{w}^{\text {neg }}\right)$. Since the upper words of $\mathbf{w}$ and $\mathbf{w}^{\text {neg }}$ are the same, the recording tableaux are the same, and hence Equation (4) holds.

Equation (3) is true since

$$
\begin{aligned}
P_{m^{*}}(\mathbf{w}) & =P_{m}\left(\mathbf{w}^{\text {inv neg inv }}\right) & & \text { by Equation }(1) \\
& =Q_{l r}\left(\mathbf{w}^{\text {inv neg }}\right) & & \text { by Proposition } 18 \\
& =Q_{m^{*}}\left(\mathbf{w}^{\text {inv }}\right) & & \text { by Equation }(4) .
\end{aligned}
$$

Equation (6) is an immediate consequence of Equation (3) and the fact that inv is an involution.

Finally, the first identity in Equation (5) follows from

$$
\begin{aligned}
Q_{m^{*}}(\mathbf{w})^{\text {neg }} & =P_{m^{*}}\left(\mathbf{w}^{\text {inv }}\right)^{\text {neg }} & & \text { by Equation }(6) \\
& =P_{l r}\left(\mathbf{w}^{\text {inv neg }}\right) & & \text { by Equation }(2) \\
& =Q_{m}\left(\mathbf{w}^{\text {inv neg inv }}\right) & & \text { by Proposition } 18
\end{aligned}
$$

and the second follows from Proposition 20. 
Theorem 22. The mapping from doubly colored biwords $\mathbf{w}$ to pairs of colored semistandard tableaux given by $P_{m^{*}}(\mathbf{w})$ and $Q_{m^{*}}(\mathbf{w})$ is a bijection. The content of the upper word is the content of $Q_{m^{*}}(\mathbf{w})$ and the content of the lower word is the content of $P_{m^{*}}(\mathbf{w})$.

Analogous to Equation (2) above, doubly mixed insertion commutes with ev.

Proposition 23. If $\mathbf{w}$ is a doubly colored biword, then

$$
P_{m^{*}}(\mathbf{w})^{\mathrm{ev}}=P_{l r}\left(\mathbf{w}^{\mathrm{ev}}\right)=P_{m^{*}}\left(\mathbf{w}^{\mathrm{ev}}\right) .
$$

Proof. The second equation is immediate from Proposition 20, since ev removes bars from the lower word. The first equation can be derived as follows:

$$
\begin{aligned}
P_{m^{*}}(\mathbf{w})^{\mathrm{ev}} & =P_{m}\left(\mathbf{w}^{\text {inv neg inv }}\right)^{\mathrm{ev}} & & \text { by Equation }(1) \\
& =P_{s}\left(\mathbf{w}^{\text {inv neg inv ev }}\right) & & \text { by Proposition } 17 \\
& =P_{s}\left(\mathbf{w}^{\text {ev inv neg inv }}\right) & & \text { by Proposition } 11 \\
& =Q_{s}\left(\mathbf{w}^{\text {ev inv neg }}\right) & & \text { by Theorem } 8 \\
& =Q_{m}\left(\mathbf{w}^{\text {ev inv }}\right) & & \text { by Proposition } 14 \\
& =P_{l r}\left(\mathbf{w}^{\text {ev }}\right) & & \text { by Proposition } 18 .
\end{aligned}
$$

Proposition 24. The operator st commutes with $P_{m^{*}}$ and $Q_{m^{*}}$.

Proof. This follows from Theorem 21, Proposition 15 and Proposition 11.

\section{Colored Words and Ribbon Tableaux}

In this section we define important classes of tableaux called domino tableaux and ribbon tableaux, and we relate these tableaux to colored words and the insertion algorithms of Haiman described in the previous section.

\subsection{Domino Tableaux}

A special kind of skew shape is a domino. This skew shape consists of two adjacent cells in the same row or same column. If they are in the same row, it is called a horizontal domino. If they are in the same column, it is called a vertical domino.

A domino tableau (resp. skew domino tableau) is a tableau (resp. skew tableau) with the following properties. First, each number appears twice in the tableau. Second, the two occurrences of each number appear adjacent to one another in the same row or in the same column. Third, the numbers weakly increase across each row and down each 
column. For example, here is a domino tableau of shape $(6,6,3,3,2)$ :

\begin{tabular}{|c|c|cc|c|c|}
\hline 1 & 2 & 4 & 4 & 7 & 8 \\
1 & 2 & 6 & 6 & 7 & 8 \\
\hline 3 & 3 & 9 & \multicolumn{2}{|l}{} \\
\cline { 1 - 2 } 5 & 5 & 9 & \multicolumn{2}{|}{} \\
\cline { 1 - 2 } 10 & 10 & \multicolumn{3}{|l}{} \\
\cline { 1 - 2 }
\end{tabular}

It is clear that the cells occupied by the same value in a domino tableau make up a domino. If $D$ is a domino tableau, then $d o m_{k}$ refers to the domino whose entries are $k$ 's, while $d o m[k]$ refers to the skew domino tableau of shape $d_{0 m}$, with entries both $k$.

Let $D o m_{\lambda}$ be the set of domino tableaux of shape $\lambda$. Note that for certain $\lambda$ (e.g., $\lambda=(3,2,1))$, this set is empty. Shapes for which $D o m_{\lambda}$ is not empty are said to have empty 2-core.

Domino tableaux are in one-to-one correspondence with pairs of standard tableaux, as described by the following theorem.

Theorem 25. There is a one-to-one correspondence between domino tableaux D, using the numbers $\{1,2, \ldots, n\}$, and pairs of standard tableaux, $(U, V)$, which together use the numbers $\{1,2, \ldots, n\}$. Furthermore, the shape of the domino tableau determines the shapes of the standard tableaux.

This bijection was probably first due to Littlewood [13], whose work was inspired by earlier papers of Robinson [18] and Nakayama [15][16]. A simple description of this bijection appears in [3] and in [5] and somewhat different descriptions appear in [26] and on page 83 of [9].

We illustrate here this bijection. Our description of the bijection follows [5]. Label each domino in $D$ either 0 or 1 according to whether the lattice distance between the upper or right cell of the domino and the main diagonal is even or odd. Similarly label each diagonal of $D$ either 0 or 1 according to whether its lattice distance to the main diagonal is even or odd.

Now delete all dominoes labeled 1 . The remaining entries on diagonals labeled 0 are the same as the entries of the diagonals of $U$. Deleting dominoes labeled 0 and retaining diagonals labeled 1 produces $V$.

In our example above, first deleting the dominoes labeled 1 gives

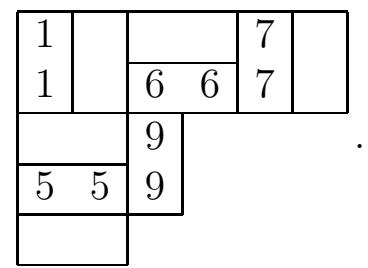

The diagonals labeled 0 then produce this tableau

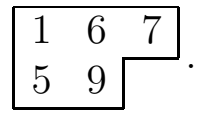

THE EleCtronic Journal of COMBinatorics 8 (2001), \#R21 
First deleting the dominoes labeled 0 gives

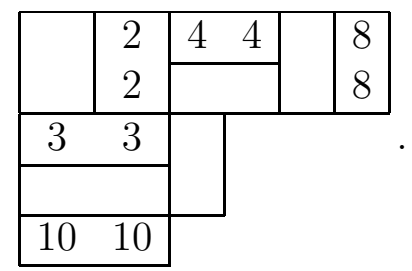

The diagonals labeled 1 then yield this tableau

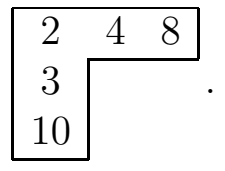

It is not too difficult to see that this is a bijection and that different domino tableaux of the same shape give the same shapes for the corresponding $U$ and $V$. We write $D=U * V$ to denote this decomposition, and $\lambda=\mu * \nu$ to denote the corresponding decomposition of the shape of $D$ into the shapes of $U$ and $V$. The pair $(U, V)$ (resp. $(\mu, \nu))$ is called the 2-quotient of $D$ (resp. $\lambda$ ).

Theorem 25 would lead one to view domino tableaux as a complicated description of a simple idea: a pair of standard Young tableaux. However, the statistic spin, defined next on domino tableaux, is not so easily described on the 2-quotient, and gives us reason to consider domino tableaux apart from their corresponding 2-quotient. See [21] for an exact description of spin on the $k$-quotient of a $k$-ribbon tableau.

For a domino (skew) tableau $D$, let ov $(D)$ be the number of vertical dominoes in odd columns and let $e v(D)$ be the number of vertical dominoes in even columns. Let $v(D)$ be the number of vertical dominoes in $D$.

For a domino (skew) tableau, $D$, spin is defined by $s p(D)=v(D) / 2$, i.e., half the number of vertical dominoes. For shape $\lambda$, let $s p^{*}$ be the maximum spin of all domino tableaux of shape $\lambda$. Then the cospin of $D$ of shape $\lambda$ is $\operatorname{cosp}(D)=s p^{*}-s p(D)$. We use cospin in this paper because of the following proposition.

Proposition 26. If $D$ is a domino tableau, then $\operatorname{cosp}(D)$ is integral.

\subsection{Ribbon Tableaux}

We now define a natural semistandard analogue of domino tableaux. Details of this construction may be found in [3].

A 2-ribbon tableau or ribbon tableau is made up of a collection of ribbons. A ribbon is the skew shape consisting of $2 k$ cells with the following property. A ribbon can be tiled by $k$ dominoes so that the cell directly above the topmost (for vertical dominoes) or rightmost (for horizontal dominoes) cell of each domino in the tiling is not in the ribbon. It is not too difficult to see that there is only one such tiling with this property. We will call this the standard 2-ribbon tiling or standard tiling. A 2-ribbon tableau $R$ has its entries weakly increasing across rows and down columns and the cells containing each 
entry form a ribbon. Since every value will appear in a ribbon tableau an even number of times, we define the content of a ribbon tableau $R$, to be the vector $\left(v_{1}, v_{2}, \ldots\right)$, where $v_{i}$ is half the number of $i$ 's appearing in $R$.

Now suppose $\lambda=\mu * \nu$. If $R$ is a ribbon tableau of shape $\lambda$, then $R$ may be decomposed into two tableaux, $U$ and $V$, of shapes $\mu$ and $\nu$ respectively, by using the domino bijection described in the previous subsection, with the dominoes determined by the standard tiling and the entries in the dominoes determined by the entries in the corresponding ribbon. The following proposition is Theorem 6.3 in [3].

Proposition 27. If the ribbon tableau $R$ corresponds to the two tableaux $U$ and $V$, then $U$ and $V$ are semistandard. Furthermore, if $U$ and $V$ are semistandard, then there is an unique ribbon tableau $R$ which corresponds to $U$ and $V$.

As with domino tableaux, we will write $R=U * V$ and we will call $(U, V)$ the 2-quotient of $R$. Define $R i b_{\lambda}$ to be the set of 2-ribbon tableaux of shape $\lambda$.

We illustrate this construction with the following example. Let $R$ be the following ribbon tableau (with the standard tiling indicated):

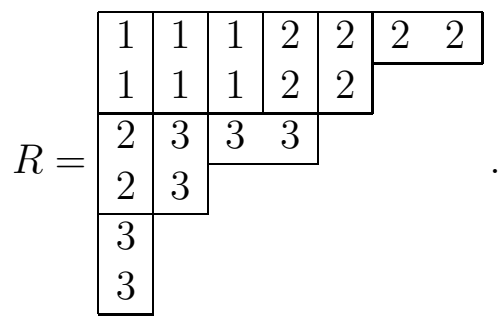

Then $U$ and $V$ are as follows.

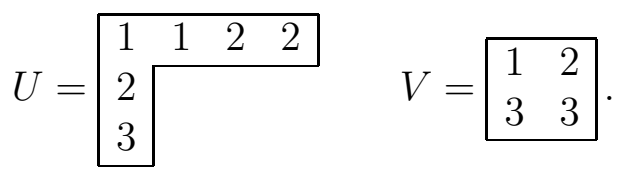

We now define spin on 2-ribbon tableaux. The spin of $R, \operatorname{sp}(R)$, is half the number of vertical dominoes in the standard 2-ribbon tiling of $R$. In the above example, $s p(R)=4$. Similarly, we can define cospin on ribbon tableaux.

Spin on 2-ribbon tableaux is discussed in [3], while spin on more general $k$-ribbon tableaux is discussed in detail in [11]. The generating function for spin on the more general ribbon tableaux generalizes the Hall-Littlewood symmetric functions [14] [11].

Finally, there is a natural standardization of ribbon tableaux. In the standard tiling of ribbon tableau $R$, within each ribbon label the dominoes in the standard tiling in increasing order from left to right. Then label the ribbons in order from smallest to largest. Since we will later view ribbon tableaux as colored tableaux, we will write $R^{\text {rst }}$ 
to denote this standardization. In the above example,

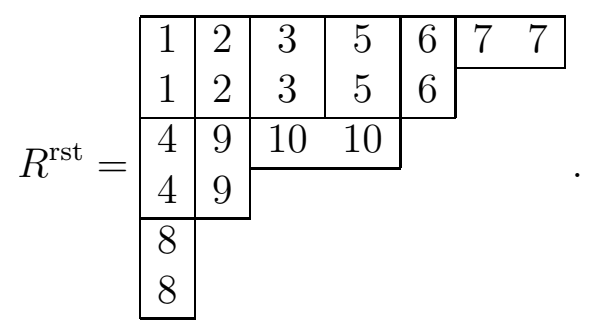

Proposition 28. Standardization is compatible with spin, that is, $s p(R)=s p\left(R^{\mathrm{rst}}\right)$. It is also compatible with the 2-quotient in the following sense: if $R=U * V$ and $R^{\mathrm{rst}}=A * B$ then $A$ and $B$ are standardizations of $U$ and $V$.

Again, using the above example, we have

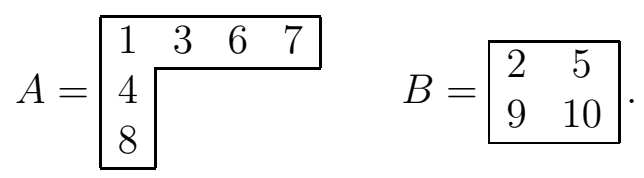

Now suppose $R$ is a 2-ribbon tableau. Following Carré and Leclerc [3], we define $w(R)$ as the column-reading word, as in the case of semistandard tableaux, except that the letter in the second occurrence of each domino is ignored. For example, if

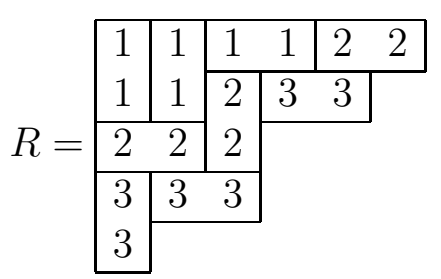

then $w(R)=321312132$. We say a 2-ribbon tableau $R$ is Yamanouchi if $w(T)$ is a reverse lattice word. For example,

$$
Y=\begin{array}{|l|l|l|ll|}
\hline 1 & 1 & 1 & 1 & 1 \\
1 & 1 & 1 & 2 & 2 \\
\hline 2 & 2 & 2 & \multicolumn{2}{|l}{} \\
\cline { 1 - 3 } 2 & 3 & 3 & \multicolumn{2}{|l}{} \\
\hline
\end{array}
$$

is a Yamanouchi 2-ribbon tableau. Unlike Yamanouchi semistandard tableaux, there can be more than one Yamanouchi 2-ribbon tableau of the same shape.

In a similar fashion we can define Yamanouchi 2-ribbon skew tableaux. The following, proved in [28], is a central result in [3].

Theorem 29. There is a bijection from 2-ribbon skew tableaux $R$ of shape $\rho / \mu$ and content $\nu$ and pairs $(Y, Q)$ where $Y$ is Yamanouchi 2-ribbon of shape $\rho / \mu$ and content $\lambda$ and $Q$ is semistandard of shape $\lambda$ and content $\nu$. Furthermore, $\operatorname{sp}(R)=s p(Y)$. 


\subsection{Domino Insertion}

In this subsection we describe a bijection from colored permutations to pairs of domino tableaux such that the total color of the permutation equals the sum of the spins of the domino tableaux. This bijection is the insertion algorithm of Garfinkle [7] and, as proved by van Leeuwen [27], is equivalent to the algorithm of Barbasch and Vogan [1].

It differs from the domino insertion in [26], which does not have the color-to-spin property. Another domino insertion is described in [22], which also has the color-to-spin property, and which extends to $k$ rim-hook tableaux. We do not use this insertion here because it does not have the necessary insertion equivalence.

In a later subsection we shall extend this bijection to 2-ribbon tableaux.

Suppose $\delta=\alpha / \beta$ is a domino. We say $\delta$ is an outer domino of $\alpha$. We will write $\alpha-\delta$ to mean $\beta$. We will also say that $\delta$ is a domino outside $\beta$. We will write $\beta+\delta$ to mean $\alpha$.

Similarly, suppose $\lambda / \mu$ is a skew shape and $\delta=\nu / \mu$ is a domino, with $\nu$ contained in $\lambda$. Then we say $\delta$ is an inner domino of $\lambda / \mu$ and we write $\lambda / \mu-\delta$ to mean $\lambda / \nu$. And we call $\delta$ a domino inside $\lambda / \nu$ and write $\lambda / \nu+\delta$ to mean $\lambda / \mu$.

If $\delta$ is a domino, let $\delta[k]$ denote the domino skew tableau of shape $\delta$ with both entries $k$.

Also, if $T$ is a domino tableau with largest entry $k$, then $d^{2} m_{k}$ is an outer domino of $\operatorname{sh}(T)$. Write $T-\operatorname{dom}[k]$ to denote the removal of this domino from $T$. Similar definitions hold for the addition of a domino to a tableau and for skew domino tableaux.

Let $\alpha$ be a shape and $\beta$ be a skew shape such that $\alpha$ and $\beta$ intersect in a domino $\delta$ which is an outer domino of $\alpha$ and an inner domino of $\beta$. We call such a pair $(\alpha, \beta)$ a domino overlapping partition pair.

Suppose $(\alpha, \beta)$ is a domino overlapping partition pair. Let $U$ be a domino tableau of shape $\alpha$ and let $V$ be a skew domino tableau of shape $\beta$. Suppose all the entries of $U$ are smaller than all the entries of $V$. We call such a pair $(U, V)$ a domino overlapping tableau pair. We say $(U, V)$ has shape $(\alpha, \beta)$.

Now suppose $(U, V)$ is a domino overlapping tableau pair of shape $(\alpha, \beta)$ with intersection $\delta$. Suppose $U$ has entries $\left\{a_{1}<a_{2}<\cdots<a_{k-1}\right\}$ and $V$ has non-empty set of entries $\left\{a_{k}<\cdots<a_{n}\right\}$. We will show how to construct another overlapping tableau pair, $(\tilde{U}, \tilde{V})$ of shape $(\tilde{\alpha}, \tilde{\beta})$ with intersection $\tilde{\delta}$, and where $\tilde{U}$ has entries $\left\{a_{1}<\cdots<a_{k}\right\}$ and $\tilde{V}$ has entries $\left\{a_{k+1}<\cdots<a_{n}\right\}$. Furthermore,

$$
s p(U)+s p(V)+s p(\delta)=s p(\tilde{U})+s p(\tilde{V})+s p(\tilde{\delta}) .
$$

We will call this algorithm Bump, that is, $(\tilde{U}, \tilde{V})=\operatorname{Bump}(U, V)$.

The construction of $\tilde{U}$ and $\tilde{V}$ proceeds by cases, depending on how $d o m_{a_{k}}$ and $\delta$ overlap. In all cases,

$$
\begin{gathered}
\tilde{\beta}=\beta-\operatorname{dom}_{a_{k}} \\
\tilde{V}=V-\operatorname{dom}\left[a_{k}\right] .
\end{gathered}
$$


If $d o m_{a_{k}}$ and $\delta$ are disjoint, then

$$
\begin{gathered}
\tilde{\alpha}=\alpha+\operatorname{dom}_{a_{k}} \\
\tilde{U}=U+\operatorname{dom}\left[a_{k}\right] \\
\tilde{\delta}=\delta .
\end{gathered}
$$

Clearly, Equation (7) holds.

If $d o m_{a_{k}}$ and $\delta$ overlap in a single cell, then one must be vertical (say $d o m_{a_{k}}$ ) and one must be horizontal (say $\delta$ ). In this case, construct a new vertical domino $\tilde{\delta}$, which will be $\delta$ with the intersecting position moved diagonally out one position. Also, construct a new horizontal domino, called $d o m_{a_{k}}^{\prime}$, from $d o m_{a_{k}}$ by moving the intersecting position diagonally out one position. Call the corresponding domino with $a_{k}$ 's $\mathrm{dom}^{\prime}\left[a_{k}\right]$. Then

$$
\begin{gathered}
\tilde{\alpha}=\alpha+\operatorname{dom}_{a_{k}}^{\prime} \\
\tilde{U}=U+\operatorname{dom}^{\prime}\left[a_{k}\right] .
\end{gathered}
$$

Note that the number of vertical dominoes in $U$ is unchanged, the number of vertical dominoes in $V$ has gone down by one, and $\delta$ has gone from horizontal to vertical. Thus, Equation (7) is established. Here is an example of this case.

$$
\begin{aligned}
& U=\begin{array}{llll}
1 & 1 & 4 & 4 \\
2 & 3 & 5 & \\
2 & 3 & 5 \\
6 & &
\end{array} \quad, \quad V=\begin{array}{|lll|}
n \\
6
\end{array}
\end{aligned}
$$

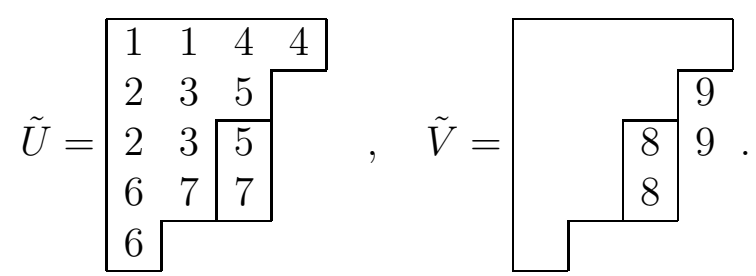

As described thus far, this algorithm is identical to the insertion algorithm of Stanton and White [26]. The difference arises when the two dominoes $d o m_{a_{k}}$ and $\delta$ are identical.

When this happens, there are two cases, depending upon whether $\delta$ is vertical or horizontal. If it is horizontal, let $\tilde{\delta}$ be the unique horizontal domino in the next row which is outside $\alpha$. Note that $\alpha+\tilde{\delta}$ is a shape, since $\delta$ was a horizontal outer domino in the previous row. Let $\tilde{\delta}\left[a_{k}\right]$ denote this domino with $a_{k}$ 's placed in it. Then define

$$
\begin{gathered}
\tilde{\alpha}=\alpha+\tilde{\delta} \\
\tilde{U}=U+\tilde{\delta}\left[a_{k}\right] .
\end{gathered}
$$


Note that the spins of $U, V$, and $\delta$ remain unchanged. Here is an example of this case.

$$
\begin{aligned}
& U=\begin{array}{llll}
1 & 1 & 4 & 4 \\
2 & 3 & 5 & \\
2 & 3 & 5 \\
6 & &
\end{array} \quad, \quad V=\begin{array}{|ll|l|}
n \\
6
\end{array} \\
& \tilde{U}=\begin{array}{llll}
1 & 1 & 4 & 4 \\
2 & 3 & 5 \\
2 & 3 & 5 \\
6 & 7 & 7 \\
& &
\end{array} \quad, \quad \tilde{V}=\begin{array}{l}
9 \\
\\
9 \\
\end{array} .
\end{aligned}
$$

The last case is when $d o m_{a_{k}}$ and $\delta$ are identical and both vertical. This case is exactly the same as the previous case, except that $\tilde{\delta}$ is the unique vertical domino in the next column which is outside $\alpha$. In this case, note that the number of vertical dominoes in $U$ goes up by 1 , the number of vertical dominoes in $V$ goes down by 1 , and both $\delta$ and $\tilde{\delta}$ are vertical. This case is illustrated below.

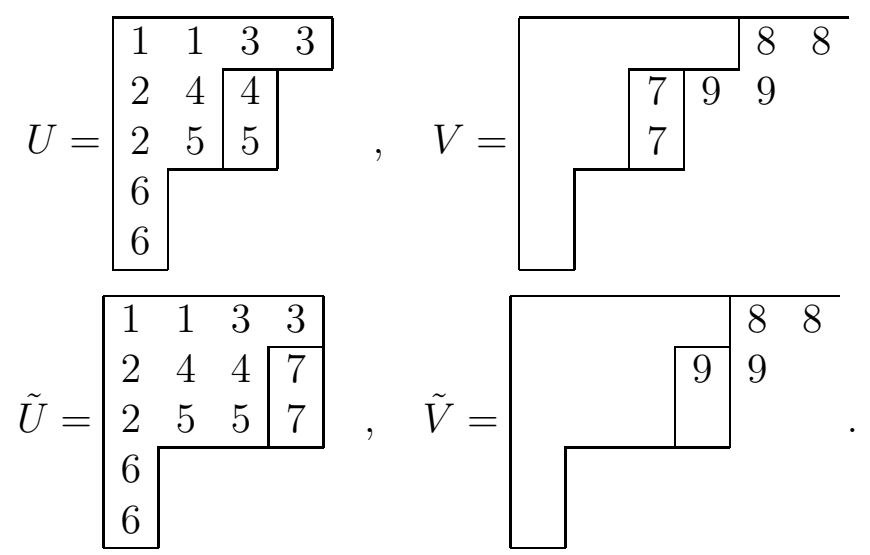

Finally, we will need a special definition to give a stopping rule. If $T$ is a domino tableau, let $\operatorname{Aug}(T)$ be a domino tableau consisting of $T$ and an outer border of domino shapes filled with letters from some larger alphabet (which we call the augmenting alphabet). For example, if

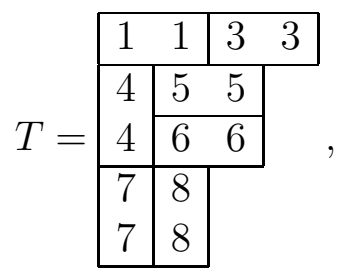


then one possibility is

$$
\operatorname{Aug}(T)=\begin{array}{|c|c|c|c|c|}
\hline 1 & 1 & 3 & 3 & \infty_{3} \\
\hline 4 & 5 & 5 & \infty_{2} & \infty_{3} \\
\cline { 2 - 4 } & 6 & 6 & \infty_{2} & \\
\hline 7 & 8 & \infty_{4} & \infty_{4} & \\
7 & 8 & \infty_{5} & \\
\hline \infty_{1} & \infty_{1} & \infty_{5} & \\
\hline
\end{array}
$$

where the augmenting alphabet is

$$
\infty_{1}<\infty_{2}<\infty_{3}<\infty_{4}<\infty_{5}
$$

Note that there are several possible choices for $A u g(T)$.

If $\lambda$ is a shape, let $\operatorname{dom}^{r}(\lambda)$ denote the domino consisting of the two cells in row 1 , columns $\lambda_{1}+1$ and $\lambda_{1}+2$. Also, if $\lambda$ has $l$ parts, let $d_{0 m^{c}}(\lambda)$ denote the domino consisting of the two cells in column 1 , rows $l+1$ and $l+2$. If $T$ is a domino tableau and $x$ is a colored letter, let $|x|$ denote the letter $x$ with the "color" removed, let $T_{<x}$ be the portion of $T$ consisting of letters smaller than $|x|$ and let $T_{>x}$ be the portion of $T$ consisting of letters larger than $|x|$.

We now describe domino insertion. Let $x$ be a colored letter and let $T$ be a domino tableau not containing $|x|$.

Algorithm 1.

if $x$ is unbarred then

$$
U:=T_{<x} \cup \operatorname{dom}^{r}\left(\operatorname{sh}\left(T_{<x}\right)\right)[|x|]
$$

else if $x$ is barred then

$$
U:=T_{<x} \cup \operatorname{dom}^{c}\left(\operatorname{sh}\left(T_{<x}\right)\right)[|x|]
$$

$W:=T_{>x}$

$V:=\operatorname{Aug}(W)$

$\{(U, V)$ forms a domino overlapping tableau pair $\}$

while $V$ contains letters not in the augmenting alphabet do

$$
(U, V):=\operatorname{Bump}(U, V)
$$

$$
(T \stackrel{\mathrm{d}}{\leftarrow} x):=U
$$

$\delta=\operatorname{sh}(T \stackrel{\mathrm{d}}{\leftarrow} x) / \operatorname{sh}(T)$

Note that from Equation (7) we have

$$
s p(T \stackrel{\mathrm{d}}{\leftarrow} x)+s p(\delta)=\left\{\begin{array}{ll}
s p(T) & \text { if } x \text { is unbarred } \\
s p(T)+1 & \text { if } x \text { is barred }
\end{array} .\right.
$$

For example, if

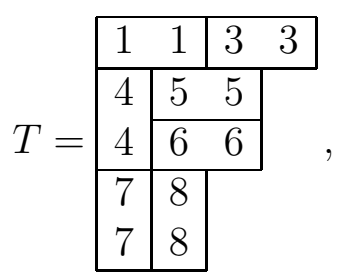


then

$$
(T \stackrel{\mathrm{d}}{\leftarrow} 2)=\begin{array}{|ll|ll|}
\hline 1 & 1 & 2 & 2 \\
\hline 3 & 3 & 5 & \\
\cline { 1 - 2 } 4 & 4 & 5 & \\
\cline { 1 - 2 } 6 & 6 & 8 & \\
\cline { 1 - 2 } 7 & 7 & 8 & \\
\hline
\end{array}
$$

The spin of $T$ is $3 / 2$ and the spin of $(T \stackrel{d}{\leftarrow} 2)$ is 1 , while the spin of $\delta$ is $1 / 2$.

As another example, if

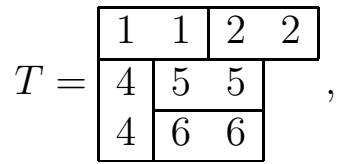

then

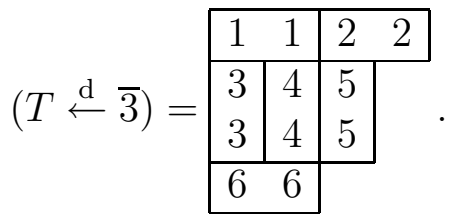

The spin of $T$ is $1 / 2$, while the spin of $(T \stackrel{d}{\leftarrow} \overline{3})$ is $3 / 2$ and the spin of $\delta$ is 0 .

We can now insert an entire colored permutation. Each application of Algorithm 1 will create a tableau which differs from the previous by a domino shape, so we can construct a recording tableau. If $\pi=a_{1} a_{2} \ldots a_{n}$ is a colored permutation, where the $a_{i}$ are colored letters, write

$$
P_{d}(\pi)=\left(\ldots\left(\left(\emptyset \stackrel{\mathrm{d}}{\leftarrow} a_{1}\right) \stackrel{\mathrm{d}}{\leftarrow} a_{2}\right) \ldots \stackrel{\mathrm{d}}{\leftarrow} a_{n}\right)
$$

and $Q_{d}(\pi)$ to represent the recording tableau.

It is clear that the algorithm described above is reversible. Equation (8) then leads to the following theorem.

Theorem 30. There is a bijection between colored permutations $\pi$ and pairs of domino tableaux of the same shape, given by

$$
P_{d}(\pi) \text { and } Q_{d}(\pi) \text {. }
$$

Furthermore,

$$
t c(\pi)=\operatorname{sp}\left(P_{d}(\pi)\right)+\operatorname{sp}\left(Q_{d}(\pi)\right) .
$$

That is, the total color of $\pi$ is the sum of the spins of the two tableaux.

We illustrate Theorem 30 with an example. Let

$$
\pi=\overline{3} 85 \overline{2} 1 \overline{9} \overline{7} 4 \overline{6} \text {. }
$$

Then

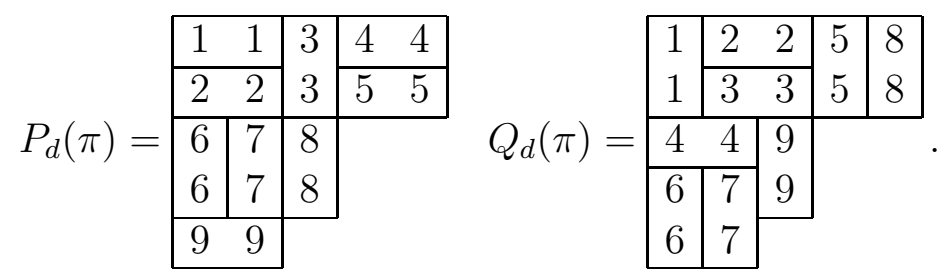

We have $t c(\pi)=5$ and $s p\left(P_{d}(\pi)\right)+s p\left(Q_{d}(\pi)\right)=2+3$. 


\subsection{Mixed Insertion and Domino Insertion}

We now draw the connection between domino insertion and doubly mixed insertion. Suppose $D$ is a domino tableau. We may regard $D$ as a colored tableau by barring exactly one number in each domino, so that the resulting tableau is standard in the alphabet of colored letters.

Lemma 31. Let $D$ be a domino tableau, let $x$ be a colored letter whose value is not in $D$, and let $x^{*}$ be the "color complement" of $x$, that is, $x^{*}$ is barred if $x$ is not and vice versa. Then

$$
\left((D \stackrel{\mathrm{m}}{\leftarrow} x) \stackrel{\mathrm{dm}}{\leftarrow} x^{*}\right)=(D \stackrel{\mathrm{d}}{\leftarrow} x) .
$$

Proof. The proof is by induction on the number of dominoes in $D$. It is easy to check that the result is true if $D$ is empty. Suppose the lemma is true for all $D$ with $k$ dominoes. Let $D$ be a domino tableau with $k+1$ dominoes. Let $z$ be the largest entry in $D$. Let $D_{0}$ denote $D$ with the domino of $z$ 's removed. First, we notice that

$$
\left.X=(D \stackrel{\mathrm{m}}{\leftarrow} x) \stackrel{\mathrm{dm}}{\leftarrow} x^{*}\right)
$$

can be done in two steps. First, we construct

$$
\left.X_{0}=\left(D_{0} \stackrel{\mathrm{m}}{\leftarrow} x\right) \stackrel{\mathrm{dm}}{\leftarrow} x^{*}\right) .
$$

By induction $\delta=\operatorname{sh}\left(X_{0}\right) / \operatorname{sh}\left(D_{0}\right)$ must be a domino. We then verify that $X$ can be constructed by first constructing $X_{0}$, and then using the two cells in $\delta$ to "bump" the domino in $D_{0}$ containing $\bar{z}$ and $z$. This is because once a particular element of $D_{0}$ has been "bumped" by the insertion of $x$, it will not be affected again until it is "bumped" by $x^{*}$.

But by induction, $X_{0}=\left(D_{0} \stackrel{\mathrm{d}}{\leftarrow} x\right)$. Thus, in the construction of $(D \stackrel{\mathrm{d}}{\leftarrow} x)$, the domino $\delta$ will "domino bump" the domino containing the $z$ 's. We must therefore verify that the result of this "domino bump" is the same as the two mixed insertion "bumps" described above. Clearly if $\delta$ does not intersect $d m_{z}$ then both processes do nothing to the $z$ 's. There are four cases left to consider. Each case has a corresponding figure. Let $a$ and $b$ be the elements bumped into $\delta$. We use $\stackrel{d}{\rightarrow}$ to indicate domino bumping and $\stackrel{m}{\rightarrow}$ and $\stackrel{d m}{\rightarrow}$ to indicate the two forms of mixed bumping.

i. The horizontal $\delta$ overlaps the vertical $d o m_{z}$. See Figure 1.

ii. The vertical $\delta$ overlaps the horizontal $d o m_{z}$. See Figure 2 .

iii. The dominoes $\delta$ and $d o m_{z}$ are vertical and equal. See Figure 3.

iv. The dominoes $\delta$ and $d o m_{z}$ are horizontal and equal. See Figure 4. 


\begin{tabular}{|c|c|c|c|c|c|}
\hline$a_{z}$ & $b$ & & & $a$ & $b$ \\
\hline$z$ & & & & $z$ & $z$ \\
\hline
\end{tabular}

\begin{tabular}{|c|c|c|c|c|c|c|c|}
\hline$a_{\bar{z}}$ & $b$ & \multirow{2}{*}{$\stackrel{m}{\longrightarrow}$} & $a$ & $b_{\bar{z}}$ & \multirow{2}{*}{$\stackrel{m^{*}}{\longrightarrow}$} & $a$ & $b$ \\
\hline$z$ & & & $z$ & & & $\bar{z}$ & $z$ \\
\hline
\end{tabular}

Figure 1: Case (i)

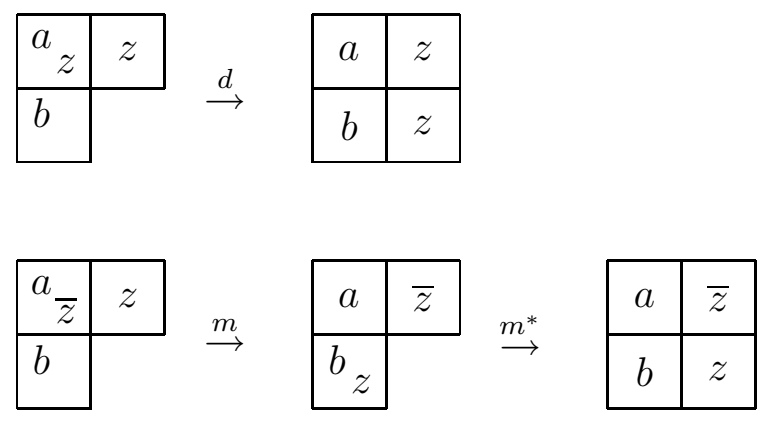

Figure 2: Case (ii)
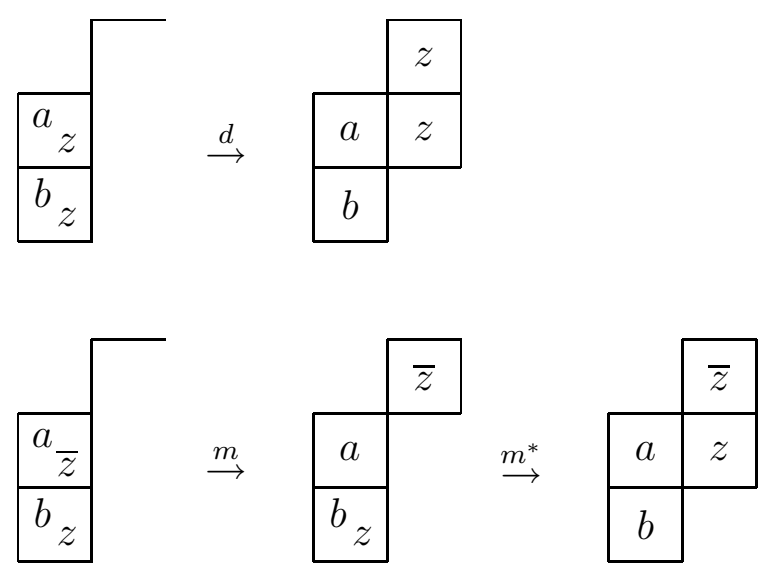

Figure 3: Case (iii) 

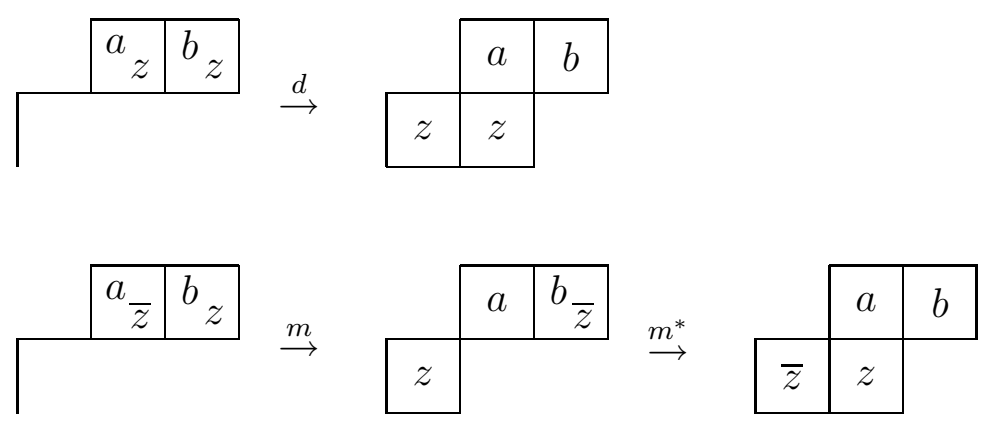

Figure 4: Case (iv)

Lemma 31 motivates the definitions of the following two operators on colored biwords. Let $\mathbf{w}$ be a colored biword. Then $\mathbf{w}^{\text {dup }}$ ("duplicate") is the doubly colored biword obtained as follows. If $k$ is a letter appearing $j$ times in the upper word of $\mathbf{w}$, then both $\bar{k}$ and $k$ will appear $j$ times in the upper word of $\mathbf{w}^{\text {dup }}$. If $\mathbf{v}$ is the subword of $\mathbf{w}$ which appears below the $k$ 's, then $\mathbf{v}$ will appear below the $k$ 's in $\mathbf{w}^{\text {dup }}$, while $\mathbf{v}^{*}$ will appear below the $\bar{k}$ 's in $\mathbf{w}^{\text {dup }}$, where $\mathbf{v}^{*}$ is $\mathbf{v}$ with the bars and non-bars reversed.

For example, if

$$
\mathbf{w}=\left(\begin{array}{lllll}
1 & 1 & 1 & 2 & 2 \\
2 & \overline{1} & 1 & \overline{2} & 3
\end{array}\right)
$$

then

$$
\mathbf{w}^{\text {dup }}=\left(\begin{array}{llllllllll}
\overline{1} & \overline{1} & \overline{1} & \frac{1}{1} & \frac{1}{1} & 1 & \overline{2} & \overline{2} & 2 & 2 \\
2 & 1 & \overline{1} & \overline{2} & \overline{1} & 1 & 2 & \overline{3} & \overline{2} & 3
\end{array}\right) .
$$

The second operator is $\mathbf{w}^{\mathrm{pal}}$ (for "palindrome"). It is simply $\mathbf{w}^{\mathrm{dup} \text { inv neg inv }}$. In the above example,

$$
\mathbf{w}^{\mathrm{pal}}=\left(\begin{array}{cccccccccc}
-2 & -2 & -1 & -1 & -1 & 1 & 1 & 1 & 2 & 2 \\
\overline{3} & 2 & \overline{1} & 1 & 2 & \overline{2} & \overline{1} & 1 & \overline{2} & 3
\end{array}\right)
$$

The colored biword $\mathbf{w}^{\mathrm{pal}}$ can be described directly. For the upper word, write the reversenegative of the upper word of $\mathbf{w}$, followed by the upper word of $\mathbf{w}$. For the lower word, write the reverse of the lower word of $\mathbf{w}$, with bars complemented, followed by the lower word of $\mathbf{w}$. This description of the lower word also defines pal on colored words.

The following theorem shows that domino insertion of $\pi$ is equivalent to doubly mixed insertion of $\pi^{\text {dup }}$ or mixed insertion of $\pi^{\text {pal }}$. It connects the Garfinkle insertion algorithm with Haiman's insertion algorithms. This theorem is essentially due to van Leeuwen [27]. 
Theorem 32. If $\pi$ is a colored permutation, then

$$
\begin{gathered}
P_{d}(\pi)=P_{m^{*}}\left(\pi^{\text {dup }}\right) \\
P_{d}(\pi)=P_{m}\left(\pi^{\text {pal }}\right) \\
Q_{d}(\pi)=Q_{m^{*}}\left(\pi^{\text {dup }}\right) \\
Q_{d}(\pi)^{\text {neg }}=Q_{m}\left(\pi^{\text {pal }}\right)
\end{gathered}
$$

Proof. The first and third identities follow immediately from Lemma 31. The second and fourth identities follow from Theorem 21.

In order to extend the domino insertion algorithm to colored biwords and ribbon tableaux, we need a lemma to replace Proposition 4. An ascent in a permutation $\pi$ is a pair of adjacent values $\pi_{i}$ and $\pi_{i+1}$ such that $\pi_{i}<\pi_{i+1}$.

Lemma 33. Let $\pi$ be a colored permutation. Then $\pi^{\text {neg }}$ has an ascent at $i$ if and only if dom $_{i}$ lies to the left of dom ${ }_{i+1}$ in $Q_{d}(\pi)$.

Proof. Let $\pi=\pi_{1} \pi_{2} \ldots \pi_{n}, \pi^{(j)}=\pi_{1} \pi_{2} \ldots \pi_{j}$ and $T^{(j)}=P_{l r}\left(\pi^{(j)^{\text {dup neg }}}\right)$. Then by Theorem 21 and Theorem $32 d o m_{j}$ in $Q_{d}(\pi)$ is the shape difference between $T^{(j-1)}$ and $T^{(j)}$. Thus,

$$
T^{(j+1)}=\left(\left(\pi_{j+1}^{*} \text { neg } \stackrel{\mathrm{s}}{\rightarrow}\left(\left(\pi_{j}^{* \text { neg }} \stackrel{\mathrm{s}}{\rightarrow} T^{j-1}\right) \stackrel{\mathrm{s}}{\leftarrow} \pi_{j}^{\mathrm{neg}}\right)\right) \stackrel{\mathrm{s}}{\leftarrow} \pi_{j+1}^{\mathrm{neg}}\right),
$$

where we again use the notation $x^{*}$ to denote the color-reversing operation. However, since row and column insertion commute (Corollary 3),

$$
T^{(j+1)}=\left(\pi_{j+1}^{*} \stackrel{\text { neg }}{\rightarrow} \stackrel{\mathrm{s}}{\rightarrow}\left(\pi_{j}^{* \text { neg }} \stackrel{\mathrm{s}}{\rightarrow}\left(\left(T^{j-1} \stackrel{\mathrm{s}}{\leftarrow} \pi_{j}^{\mathrm{neg}}\right) \stackrel{\mathrm{s}}{\leftarrow} \pi_{j+1}^{\mathrm{neg}}\right)\right)\right) .
$$

The insertion of $\pi_{j}^{\text {neg }}$ into $T$ starts the domino $d o m_{j}$, while the subsequent insertion of $\pi_{j+1}^{\text {neg }}$ starts the domino $d o m_{j+1}$. The result then follows from Proposition 4.

If $\mathbf{w}$ is a colored biword whose lower word is standard, then $P_{d}(\mathbf{w})$ is still defined and is a domino tableau. It follows from Lemma 33 that $Q_{d}(\mathbf{w})$, formed by recording the upper letter in the domino shape created at each step of domino insertion, is a ribbon tableau.

Corollary 34. If $\mathbf{w}$ is a colored biword whose lower word uses a standard alphabet, then $Q_{d}(\mathbf{w})$ is a ribbon tableau and, in fact,

$$
Q_{d}\left(\mathbf{w}^{\overline{\mathrm{st}}}\right)=Q_{d}(\mathbf{w})^{\mathrm{rst}}
$$

\section{A Ribbon RSK-Correspondence}

In this section we describe a RSK-correspondence for colored biwords and pairs of ribbon tableaux. We then describe its relationship to the $q$-Littlewood-Richardson coefficients of Carré and Leclerc. 


\subsection{The Ribbon RSK-Correspondence}

While it would be possible to define an RSK-correspondence, similar to that described in Algorithm 1, which maps colored biwords directly into pairs of ribbon tableaux, it is more convenient at this point to use the connection to doubly mixed insertion given by Theorem 32 and our standardization results.

Motivated by Theorem 32, we define $P_{r}$ and $Q_{r}$ on colored biwords as follows:

$$
P_{r}(\mathbf{w})=P_{m^{*}}\left(\mathbf{w}^{\text {dup }}\right)
$$

and

$$
Q_{r}(\mathbf{w})=Q_{m^{*}}\left(\mathbf{w}^{\text {dup }}\right) .
$$

Note that $P_{r}(\mathbf{w})$ and $Q_{r}(\mathbf{w})$ are colored tableaux. The tableaux $P_{d}(\mathbf{w})$ and $P_{r}(\mathbf{w})$ agree on colored permutations (except one is domino and the other colored) as do $Q_{d}(\mathbf{w})$ and $Q_{r}(\mathbf{w})$.

We will eventually show that $P_{r}(\mathbf{w})$ and $Q_{r}(\mathbf{w})$ may be regarded as ribbon tableaux. But first, we describe several of the properties of these tableaux, based on Theorem 21 .

Let $\mathbf{w}$ be a colored biword. We define a new "inverse" operator $\mathbf{w}^{\text {inv }_{\mathbf{r}}}$ to be $\mathbf{w}^{\text {inv }}$, except that the colors are placed on the lower letter of each biletter. In fact, if we define the operator up on colored biwords $\mathbf{w}$ so that $\mathbf{w}^{\mathrm{up}}$ moves the bars from the lower letter to the upper letter on each biletter, then $\mathbf{w}^{\text {inv }_{\mathbf{r}}}=\mathbf{w}^{\text {upinv }}$. For example, if

$$
\mathbf{w}=\left(\begin{array}{llllll}
1 & 1 & 1 & 2 & 2 & 3 \\
\overline{2} & 1 & 2 & \overline{1} & 3 & \overline{1}
\end{array}\right)
$$

then

$$
\mathbf{w}^{\mathrm{up}}=\left(\begin{array}{cccccc}
\overline{1} & 1 & 1 & \overline{2} & 2 & \overline{3} \\
2 & 1 & 2 & 1 & 3 & 1
\end{array}\right)
$$

and

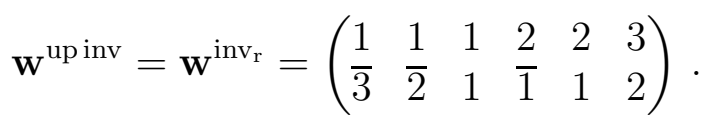

The definitions of colored biword and doubly colored biword guarantee that $\mathbf{w}^{\text {inv }_{\mathbf{r}}}$ will be a colored biword.

Proposition 35. The operator inv $\mathrm{r}_{\mathrm{r}}$ commutes with dup and ev in the following sense.

$$
\mathbf{w}^{\mathrm{inv}_{\mathrm{r}} \mathrm{ev}}=\mathbf{w}^{\mathrm{evinv}}
$$

and

$$
\mathbf{w}^{\text {inv }_{\mathrm{r}} \text { dup }}=\mathbf{w}^{\text {dup inv }} .
$$

Proposition 36. If a colored biword is duplicated, then evacuated, the result is that the bars migrate to the upper word. That is, $\mathbf{w}^{\text {dupev }}=\mathbf{w}^{\text {up }}$. 
Theorem 37. If $\mathbf{w}$ is a colored biword, then we have

$$
\begin{gathered}
P_{r}(\mathbf{w})=P_{m}\left(\mathbf{w}^{\mathrm{pal}}\right) \\
Q_{r}(\mathbf{w})^{\mathrm{neg}}=Q_{m}\left(\mathbf{w}^{\mathrm{pal}}\right) \\
P_{r}(\mathbf{w})=Q_{r}\left(\mathbf{w}^{\text {inv }_{\mathrm{r}}}\right) \\
Q_{r}(\mathbf{w})=P_{r}\left(\mathbf{w}^{\mathrm{inv}_{\mathrm{r}}}\right) \\
P_{r}(\mathbf{w})^{\mathrm{ev}}=P_{l r}\left(\mathbf{w}^{\mathrm{up}}\right) \\
Q_{r}(\mathbf{w})^{\mathrm{ev}}=Q_{m}(\mathbf{w})=Q_{s}\left(\mathbf{w}^{\mathrm{neg}}\right)
\end{gathered}
$$

The permutation version of Equation (11) and Equation (12) was given in [27].

Proof. Equation (9) and Equation (10) follow directly from Theorem 21. Equation (11) is a consequence of Theorem 21 and Proposition 35. Equation (12) follows from the involutionary property of inv $_{\mathrm{r}}$ and from Equation (11). Equation (13) follows from Proposition 23 and Proposition 36. Finally, Equation (14) is proved as follows.

$$
\begin{aligned}
& Q_{r}(\mathbf{w})^{\mathrm{ev}}=P_{r}\left(\mathbf{w}^{\mathrm{inv}{ }_{\mathrm{r}}}\right)^{\mathrm{ev}} \quad \text { by Equation (12) } \\
& =P_{l r}\left(\mathbf{w}^{\text {inv }_{\mathrm{r}} \text { up }}\right) \quad \text { by Equation (13) } \\
& =P_{l r}\left(\mathbf{w}^{\text {inv }}\right) \\
& =Q_{m}(\mathbf{w}) \quad \text { by Proposition } 18 \\
& =Q_{s}\left(\mathbf{w}^{\text {neg }}\right) \quad \text { by Proposition } 14 \text {. }
\end{aligned}
$$

It follows from Equation (9) that $P_{r}(\mathbf{w})$ is independent of its upper word.

Suppose $R$ is a ribbon tableaux. We define an operator $R^{\text {neg }}$ similar to the neg operator on colored tableaux. Working from the smallest ribbon to largest, and within a ribbon from left to right, convert (using the conversion operation described in Subsection 3.2) exactly one of the pair corresponding to one of the dominoes in the standard tiling to its negative. If $R$ is a domino tableau, then this operation is just the usual $R^{\text {neg }}$ where $R$ is regarded as a colored tableau, with each domino having exactly one barred letter and one unbarred letter. This operation is compatible with ribbon standardization in the following sense:

$$
R^{\text {rst neg }}=R^{\text {neg st }} .
$$

Now suppose $T$ is a colored tableau such that there is a ribbon tableau $R$ satisfying $R^{\text {neg }}=T^{\text {neg }}$. The invertibility of neg establishes the uniqueness of $R$. We will therefore regard $R$ and $T$ as the same tableau and call $T$ a ribbon tableau, even though technically it is not. Note that $R$ can be recovered from $T$ by simply removing the bars.

For example, let

$$
T=\begin{array}{llllllll|}
\overline{1} & \overline{1} & \overline{1} & 1 & \overline{2} & \overline{2} & 2 & 2 \\
1 & 1 & \overline{2} & & & & & \\
\overline{2} & 2 & 2 & & & & & \\
\hline
\end{array}
$$


and

$$
R=\begin{array}{llllllll}
1 & 1 & 1 & 1 & 2 & 2 & 2 & 2 \\
\cline { 3 - 6 } & 1 & 2 & & & & & \\
2 & 2 & 2 & & & & &
\end{array} .
$$

Then we have

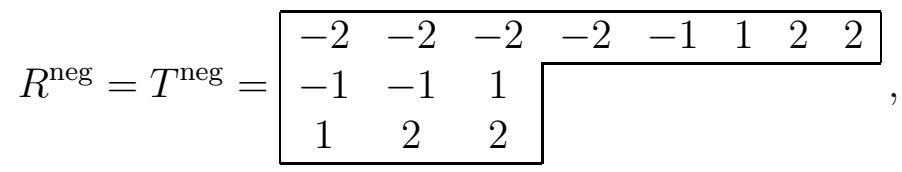

and we regard $T$ and $R$ as the same tableau, that is, both ribbon and colored.

Lemma 38. If $\mathbf{w}$ is a colored biword, then both $P_{r}(\mathbf{w})$ and $Q_{r}(\mathbf{w})$ are ribbon tableaux (in the sense defined above).

Proof. We first show that if $\mathbf{w}$ has a standard lower word, then $Q_{r}(\mathbf{w})$ is ribbon. The tableau $Q_{d}(\mathbf{w})$ is ribbon (Corollary 34), so we will show $Q_{r}(\mathbf{w})=Q_{d}(\mathbf{w})$. First form $Q_{d}(\mathbf{w})^{\text {negst }}$, that is, negate as described above, then standardize. By Equation (15) this is $Q_{d}(\mathbf{w})^{\text {rst neg }}$. From Corollary 34, this is $Q_{d}\left(\mathbf{w}^{\mathrm{st}}\right)^{\text {neg }}$. Since $Q_{d}$ and $Q_{r}$ agree on colored permutations and $\mathbf{w}^{\overline{s t}}$ is a colored permutation, we have

$$
Q_{d}\left(\mathbf{w}^{\overline{\mathrm{st}}}\right)^{\mathrm{neg}}=Q_{r}\left(\mathbf{w}^{\overline{\mathrm{st}}}\right)^{\mathrm{neg}} .
$$

By Theorem 37, this is $Q_{m}\left(\mathbf{w}^{\overline{\text { st }}}\right.$ pal $)$, which is also $Q_{m}\left(\mathbf{w}^{\text {palst }}\right)$, from the obvious fact that standardization commutes with pal on colored biwords with standard lower word. But st commutes with $Q_{m}$ by Proposition 15, so we have

$$
Q_{d}(\mathbf{w})^{\mathrm{negst}}=Q_{m}\left(\mathbf{w}^{\mathrm{pal}}\right)^{\mathrm{st}} .
$$

Since $Q_{d}(\mathbf{w})^{\text {neg }}$ and $Q_{m}\left(\mathbf{w}^{\mathrm{pal}}\right)$ both use the same alphabet, the fact that their standardizations are equal means they are equal. By Theorem 37,

$$
Q_{m}\left(\mathbf{w}^{\mathrm{pal}}\right)=Q_{r}(\mathbf{w})^{\mathrm{neg}}
$$

and the result follows by the invertibility of neg.

Now we extend to arbitrary colored biwords. We first show $P_{r}(\mathbf{w})$ is ribbon. Since $P_{r}(\mathbf{w})$ is independent of the upper word, $P_{r}(\mathbf{w})=P_{r}\left(\mathbf{w}^{\overline{\mathrm{st}}}\right)$. But by Theorem 37, this is $Q_{r}\left(\mathbf{w}^{\overline{\text { stinv }_{\mathrm{r}}}}\right)$. However, $\mathbf{w}^{\overline{\mathrm{st}_{\mathrm{inv}}}}$ has standard lower word, and so this tableau is ribbon.

It then follows that $Q_{r}(\mathbf{w})$ will be ribbon by Equation (12).

Proposition 39. The ribbon RSK-algorithm commutes with standardization. That is, for colored biword $\mathbf{w}$,

$$
Q_{r}(\mathbf{w})^{\mathrm{rst}}=Q_{r}\left(\mathbf{w}^{\mathrm{st}}\right)
$$

and

$$
P_{r}(\mathbf{w})^{\mathrm{rst}}=P_{r}\left(\mathbf{w}^{\mathrm{st}}\right) .
$$


Proof. It is easy to check that $\mathbf{w}^{\overline{\mathrm{st} i n v_{\mathrm{r}}} \overline{\mathrm{st}}}=\mathbf{w}^{\text {stinv }_{\mathrm{r}}}$ for colored biwords $\mathbf{w}$. Therefore we have

$$
\begin{aligned}
P_{r}(\mathbf{w})^{\mathrm{rst}} & =P_{r}\left(\mathbf{w}^{\overline{\mathrm{st}}}\right)^{\mathrm{rst}} & & \\
& =Q_{r}\left(\mathbf{w}^{\overline{\mathrm{st}} \operatorname{inv}_{\mathrm{r}}}\right)^{\mathrm{rst}} & & \text { by Theorem } 37 \\
& =Q_{r}\left(\mathbf{w}^{\overline{\mathrm{st}^{\mathrm{inv}} \mathrm{st}}}\right) & & \text { by Corollary } 34 \\
& =Q_{r}\left(\mathbf{w}^{\mathrm{stinv}_{\mathrm{r}}}\right) & & \text { from above } \\
& =P_{r}\left(\mathbf{w}^{\mathrm{st}}\right) & & \text { by Theorem } 37 .
\end{aligned}
$$

The calculation for $Q_{r}$ is similar.

Theorem 40. If $\mathbf{w}$ is a colored biword, then the two tableaux $P_{r}(\mathbf{w})$ and $Q_{r}(\mathbf{w})$ are ribbon tableaux. Furthermore, this mapping is a bijection from colored biwords to pairs of ribbon tableaux. The content of $P_{r}(\mathbf{w})$ is the content of the lower word in $\mathbf{w}$, while the content of $Q_{r}(\mathbf{w})$ is the content of the upper word in $\mathbf{w}$. Finally,

$$
t c(\mathbf{w})=s p\left(P_{r}(\mathbf{w})\right)+s p\left(Q_{r}(\mathbf{w})\right) .
$$

Proof. That the contents are as described is immediate from the definition. The tableaux are ribbon from Lemma 38. Finally, the color-to-spin property follows from Proposition 39 and Theorem 30 .

We illustrate Theorem 40 with the following example. Suppose

$$
\mathbf{w}=\left(\begin{array}{lllllllll}
1 & 1 & 1 & 2 & 2 & 2 & 3 & 3 & 3 \\
1 & 2 & 3 & \overline{3} & \overline{1} & 2 & \overline{2} & \overline{2} & 1
\end{array}\right)
$$

Then

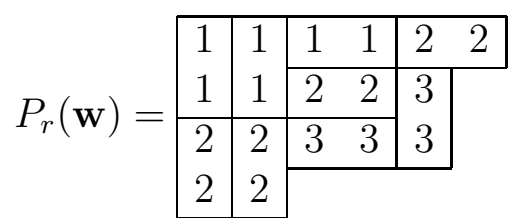

and

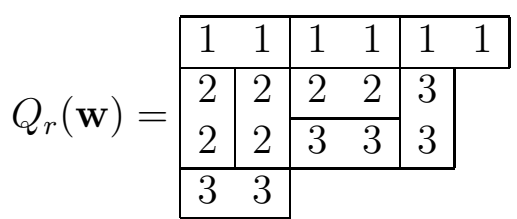

Note that $t c(\mathbf{w})=4$ while $\operatorname{sp}\left(P_{r}(\mathbf{w})\right)+\operatorname{sp}\left(Q_{r}(\mathbf{w})=5 / 2+3 / 2\right.$.

Recall that $P_{r}(\mathbf{w})$ is independent of the upper word. We may therefore speak of "ribbon insertion" of colored words, and define ribbon equivalence. That is, we say two colored words $\mathbf{w}$ and $\mathbf{v}$ are ribbon equivalent if $P_{r}(\mathbf{w})=P_{r}(\mathbf{v})$ and we write $\mathbf{w} \stackrel{\mathrm{r}}{\sim} \mathbf{v}$.

Theorem 41. If $\mathbf{w}$ and $\mathbf{v}$ are two colored words, then $\mathbf{w} \stackrel{\mathrm{r}}{\sim} \mathbf{v}$ if and only if $\mathbf{w}^{\text {palneg }} \stackrel{\mathrm{s}}{\sim}$ $\mathbf{v}^{\text {pal neg. }}$. 
Proof. This result is immediate from Theorem 37 and Corollary 16.

Theorem 41 has two corollaries which will play a significant role in Section 6 .

Corollary 42. If $\mathbf{w}$ and $\mathbf{v}$ are two colored words and if $\mathbf{w}^{\text {neg }} \stackrel{\mathrm{s}}{\sim} \mathbf{v}^{\text {neg }}$ then $\mathbf{w} \stackrel{\mathrm{r}}{\sim} \mathbf{v}$.

Proof. Let $\mathbf{w}^{\prime}$ be the colored word obtained from $\mathbf{w}$ by reversing and swapping bars with non-bars. Thus $\mathbf{w}^{\mathrm{pal}}=\mathbf{w}^{\prime} \mathbf{w}$. If $\mathbf{w}^{\text {neg }} \stackrel{\mathrm{s}}{\sim} \mathbf{v}^{\text {neg }}$, then by the definition of Knuth equivalence, $\mathbf{w}^{\prime \text { neg }} \stackrel{\stackrel{s}{\sim}}{\sim} \mathbf{v}^{\text {'neg }}$. Therefore, $\mathbf{w}^{\text {pal neg }} \stackrel{\mathrm{s}}{\sim} \mathbf{v}^{\text {pal neg }}$, so by Theorem $41, \mathbf{w} \stackrel{\mathrm{r}}{\sim} \mathbf{v}$.

Corollary 43. Let $\mathbf{w}$ be a colored word with $\mathbf{w}=\bar{k} w_{2} \ldots w_{n}$, where the $w_{i}$ are colored letters. Let $\mathbf{v}=k w_{2} w_{3} \ldots w_{n}$. Then $\mathbf{w} \stackrel{\mathrm{r}}{\sim} \mathbf{v}$ if any of the follow three conditions holds.

i. $w_{2}=j$ and $k>j$;

ii. $w_{2}=\bar{j}$ and $k>j$;

iii. $w_{2}=\bar{k}$.

Proof. In the first case, we have

$$
\mathbf{w}^{\mathrm{palneg}}=\cdots-j k-k j \cdots
$$

and

$$
\mathbf{v}^{\text {pal neg }}=\cdots-j-k k j \cdots .
$$

In the second case,

$$
\mathbf{w}^{\text {pal neg }}=\cdots j k-k-j \cdots
$$

and

$$
\mathbf{v}^{\text {pal neg }}=\cdots j-k k-j \cdots .
$$

And in the third case

$$
\mathbf{w}^{\mathrm{pal} \mathrm{neg}}=\cdots k k-k-k \cdots
$$

and

$$
\mathbf{v}^{\text {pal neg }}=\cdots k-k k-k \cdots .
$$

In all three cases, $\mathbf{w}^{\text {pal neg }} \stackrel{\mathrm{s}}{\sim} \mathbf{v}^{\text {palneg }}$, so by Theorem $41, \mathbf{w} \stackrel{\text { r }}{\sim} \mathbf{v}$.

\section{$5.2 q$-Littlewood-Richardson Coefficients}

We will now consider the generating function for cospin for 2-ribbon tableaux. Suppose $R$ is a 2-ribbon tableau, with $R=U * V$. Let $\mathbf{x}=\left\{x_{1}, x_{2}, \ldots\right\}$ be a set of indeterminates. Define

$$
\mathbf{x}^{R}=\mathbf{x}^{U} \mathbf{x}^{V} .
$$

For a shape $\rho$, define

$$
\mathcal{G}_{\rho}(\mathbf{x} ; q)=\sum_{R \in R i b_{\rho}} \mathbf{x}^{R} q^{\cos p(R)} .
$$

The following theorem is due to Carré and Leclerc [3]. 
Theorem 44. For partition $\rho=\mu * \nu$,

$$
\mathcal{G}_{\rho}(\mathbf{x} ; q)=\sum_{\lambda} c_{\mu, \nu}^{\lambda}(q) s_{\lambda}(\mathbf{x})
$$

where $c_{\mu, \nu}^{\lambda}(q)$ is a polynomial in $q$ with non-negative integer coefficients and $c_{\mu, \nu}^{\lambda}(1)=c_{\mu, \nu}^{\lambda}$.

We will call the coefficients $c_{\mu, \nu}^{\lambda}(q) q$-Littlewood-Richardson coefficients. Our $q$-LittlewoodRichardson coefficients (and our generating function $\mathcal{G}_{\rho}(\mathbf{x} ; q)$ ) differ from those of Carré and Leclerc in that we use cospin instead of spin. Our preference for cospin stems from the fact that cospin is integral, while spin is not necessarily so.

The generating function for spin on more general ribbon tableaux generalizes the HallLittlewood symmetric functions [14] [11].

Fix once and for all a ribbon tableaux $R$ of shape $\rho=\mu * \nu$. We now consider all $T \in R i b_{\rho}$ and the colored biwords $\mathbf{w}_{T}$ formed through Theorem 40 so that $P_{r}\left(\mathbf{w}_{T}\right)=R$ and $Q_{r}\left(\mathbf{w}_{T}\right)=T$. Now form the two semistandard tableaux obtained by applying the usual RSK-correspondence to $\mathbf{w}_{T}^{\text {neg }}, P_{T}=P_{s}\left(\mathbf{w}_{T}^{\text {neg }}\right)$ and $Q_{T}=Q_{s}\left(\mathbf{w}_{T}^{\text {neg }}\right)$. As $T$ ranges over all of $R i b_{\rho}, P_{T}$ will range over a certain set of semistandard tableaux. These tableaux will have positive and negative entries, and the number of negative entries, which we will call $t c\left(P_{T}\right)$, will be the same as $t c\left(\mathbf{w}_{T}\right)$.

Furthermore, since Knuth equivalence on the negative of colored words implies ribbon equivalence on the colored words, for each such $P_{T}$, every possible semistandard tableau of the same shape will appear as a possible $Q_{T}$.

Theorem 45. Let $R$ be a fixed ribbon tableau of shape $\rho=\mu * \nu$ and let $T$ range over $R i b_{\rho}$. Let $\mathbf{w}_{T}, P_{T}$ and $Q_{T}$ be as described above. For each $\lambda$ and for each $Q_{T} \in S S_{\lambda}$ there are exactly $c_{\mu, \nu}^{\lambda}$ corresponding $P_{T}$. The content of $Q_{T}$ is the content of $T$. The sum of the spins of $R$ and $T$ equals the number of minus signs in $P_{T}$. In fact,

$$
\sum_{P_{T}} q^{r_{\rho}-t c\left(P_{T}\right)}=q^{\operatorname{cosp}(R)} c_{\mu, \nu}^{\lambda}(q)
$$

where the sum is over all such $P_{T}$ of shape $\lambda$, and where $r_{\rho}=2 s p^{*}$, that is, the maximum number of vertical dominoes in a domino tableau of shape $\rho$.

Proof. The spin-to-color property of the ribbon RSK algorithm and the fact that every possible $Q_{T}$ must occur gives us

$$
\begin{aligned}
q^{s p(R)} \sum_{T \in R i b_{\rho}} q^{s p(T)} \mathbf{x}^{T} & =\sum_{\lambda} \sum_{P_{T}} \sum_{Q_{T} \in S S_{\lambda}} q^{t c\left(P_{T}\right)} \mathbf{x}^{Q} \\
& =\sum_{\lambda} s_{\lambda}(\mathbf{x}) \sum_{P_{T}} q^{t c\left(P_{T}\right)}
\end{aligned}
$$

Now converting to cospin gives

$$
q^{\operatorname{cosp}(R)} \sum_{T \in R i b_{\rho}} q^{\cos p(T)} \mathbf{x}^{T}=\sum_{\lambda} s_{\lambda}(\mathbf{x}) \sum_{P_{T}} q^{r_{\rho}-t c\left(P_{T}\right)} .
$$


But by Theorem 44,

$$
q^{\cos p(R)} \sum_{T \in R i b_{\rho}} q^{\operatorname{cosp}(T)} \mathbf{x}^{T}=q^{\cos p(R)} \sum_{\lambda} c_{\mu, \nu}^{\lambda}(q) s_{\lambda}(\mathbf{x})
$$

and the result follows by equating coefficients of $s_{\lambda}$.

To illustrate, suppose $\rho=(4,4,4,2,2,2), \mu=(2,1,1), \nu=(2,2,1)$, so that $\rho=\mu * \nu$. Then if $\lambda=(4,3,1,1)$ we have that $c_{\mu, \nu}^{\lambda}=1$. Now pick any $R$ of shape $\rho$, say

$$
R=\begin{array}{|l|l|l|l|}
\hline 1 & 1 & 2 & 2 \\
\hline 2 & 2 & 3 & 4 \\
2 & 2 & 3 & 4 \\
\hline 4 & 4 & \multicolumn{3}{|l}{} \\
\cline { 1 - 2 } 5 & 5 & \multicolumn{3}{|l}{} \\
5 & 5 & \multicolumn{3}{|l}{} \\
\cline { 1 - 1 }
\end{array}
$$

Then there is a unique $T$, which in this case is

$$
T=\begin{array}{|l|l|l|l|}
\hline 1 & 1 & 1 & 4 \\
1 & 1 & 1 & 4 \\
\hline 2 & 2 & 5 & 5 \\
\hline 3 & 3 & \multicolumn{3}{|l}{} \\
3 & 3 & \multicolumn{3}{|l}{} \\
\cline { 1 - 2 } 4 & 4 & \multicolumn{2}{|l}{} \\
\hline
\end{array}
$$

such that $P_{T}$ has shape $\lambda$. In fact, we have

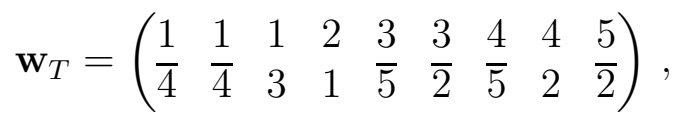

$$
\begin{aligned}
& P_{T}=\begin{array}{cccc}
-5 & -5 & -2 & -2 \\
-4 & -4 & 2
\end{array} \quad \text { and } \quad Q_{T}=\begin{array}{lllll}
1 & 1 & 1 & 4 \\
2 & 3 & 5 & \\
3 & & & &
\end{array}
\end{aligned}
$$

Note that $T$ and $R$ both have spin 3 , and $P_{T}$ has 6 minus signs.

The fact that the $c_{\mu, \nu}^{\lambda}(q)$ are polynomials in $q$ with non-negative integer coefficients is an immediate consequence of this proof. Also immediate is the fact that $\mathcal{G}_{\rho}(\mathbf{x} ; q)$ is a symmetric function. This can be realized combinatorially by converting $T$ to $\left(P_{T}, Q_{T}\right)$, then applying a multiplicity swapping bijection to $Q_{T}$, then reversing the bijection. Multiplicity swapping on semistandard tableaux is usually done in one of two ways. One, so-called Knuth switching, is due to Knuth [10], but can also be viewed as jeu de taquin. The other, the so-called automorphism of conjugation [12], results in an $S_{n}$ action on the tableaux.

By setting $q=1$ in Theorem 45 we obtain the following corollary which describes a new Littlewood-Richardson rule. 
Corollary 46. Let $R$ be a ribbon tableau of shape $\rho=\mu * \nu$. Then

$$
\#\left\{P_{T}: \operatorname{sh}\left(P_{T}\right)=\lambda\right\}=c_{\mu, \nu}^{\lambda} .
$$

We can short-circuit the calculation of $Q_{T}$ as follows.

Corollary 47. The tableau $Q_{T}$ is independent of $R$ and in fact

$$
Q_{T}=T^{\mathrm{ev}} .
$$

Proof. This is immediate from Theorem 45 and Theorem 37.

Our construction is related to the construction of Carré and Leclerc (Theorem 29) by the following theorem.

Theorem 48. Let $T$ be a 2-ribbon tableau of shape $\rho$. Then under the bijection in Theorem 29, T maps to $(Y, Q)$ where $Q=Q_{T}$ has shape $\lambda, Q_{Y}$ is Yamanouchi of shape $\lambda$, and $P_{Y}=P_{T}$.

The fact that the $Q$ in the Carré-Leclerc bijection is exactly $T^{\mathrm{ev}}$ is due to van Leeuwen [28].

We now characterize colored words whose ribbon insertion tableau is Yamanouchi.

Theorem 49. Let $\mathbf{w}$ be a colored word. Then $P_{r}(\mathbf{w})$ is Yamanouchi if and only if $\mathbf{w}^{\mathrm{pal} e v}$ is a reverse lattice word.

Proof. We replace $\mathbf{w}$ with a corresponding colored biword: $\mathbf{w}$ is the bottom row and the top row is standard. Call this colored biword also w. A result of van Leeuwen [28] states that the ribbon tableau $T$ is Yamanouchi if and only if $T^{\mathrm{ev}}$ is Yamanouchi. But $P_{r}(\mathbf{w})^{\mathrm{ev}}=P_{s}\left(\mathbf{w}^{\text {palev }}\right)$ by Proposition 17 and Theorem 37 , and by Proposition $6 P_{s}\left(\mathbf{w}^{\mathrm{palev}}\right)$ is Yamanouchi if and only if $\mathbf{w}^{\mathrm{palev}}$ is a reverse lattice word.

We illustrate with the following example. Let

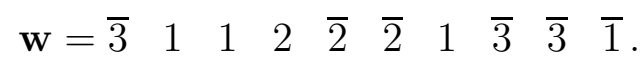

Then

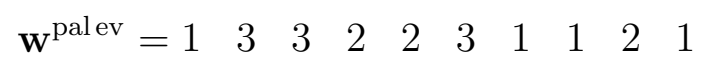

and

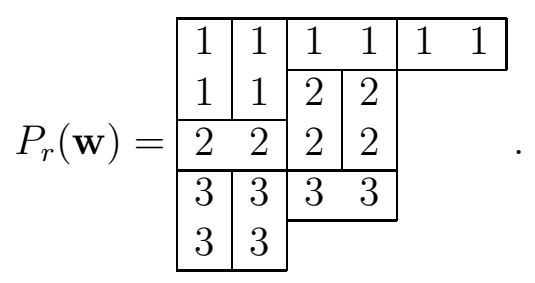

Note that $P_{r}(\mathbf{w})$ is Yamanouchi while $\mathbf{w}^{\text {pal ev }}$ is a reverse lattice word. 


\section{An Application to Rectangles}

In this section we analyze the $q$-Littlewood-Richardson coefficients of Subsection 5.2 for ribbon tableaux whose 2-quotient is a pair of rectangular shapes.

\section{1 d-Rectangles and Semi-Self-Complementary Shapes}

Let $\rho$ be the partition whose 2-quotient is a pair of rectangles $\alpha$ and $\beta$. That is, $\rho=\alpha * \beta$ with $\alpha=l^{k}$ and $\beta=n^{m}$. We say $\rho$ is $(\alpha, \beta) d$-rectangular, or simply d-rectangular. For instance, the shape $(7,6,6,5)$ is $\left(4^{2}, 2^{2}\right)$ d-rectangular.

Suppose $\beta=n^{m}$ is a rectangle and suppose $\lambda \subseteq \beta$, where

$$
\lambda=\left(\lambda_{1}, \lambda_{2}, \ldots, \lambda_{m}\right) .
$$

We call the partition $\lambda^{c}$ the $\beta$-complement of $\lambda$ if

$$
\lambda^{c}=\left(n-\lambda_{m}, n-\lambda_{m-1}, \ldots, n-\lambda_{1}\right) .
$$

We say $\lambda$ is $\beta$ self-complementary if $\lambda^{c}=\lambda$.

For example, if $\beta=(5,5,5,5)$ and $\lambda=(4,2,1,0)$, then $\lambda^{c}=(5,4,3,1)$. Also, $(4,4,1,1)$ is $\beta$ self-complementary.

Semi-self-complementary partitions are a generalization of self-complementary partitions. They depend upon two rectangles.

Let $\alpha$ and $\beta$ be two rectangles. Let $\gamma$ be the cellwise union of the Ferrers diagrams of $\alpha$ and $\beta$. We call the shape $\lambda(\alpha, \beta)$ semi-self-complementary if

i. The partition $\lambda$ contains the cells in $\gamma$.

ii. The skew shape $\lambda / \gamma$ consists of at most two shapes, one $(\mu)$ to the right of $\gamma$ and one $(\nu)$ below $\gamma$.

iii. The shapes $\mu$ and $\nu$ are $\alpha \cap \beta$-complementary.

Here are some examples of semi-self-complementary shapes. If $\alpha=6^{4}$ and $\beta=4^{5}$, then let $\lambda=(10,8,7,6,4,4,3,2,0)$ with $\mu=(4,2,1,0)$ and $\nu=(4,3,2,0)$. Figure 5 shows the shape $\lambda$ with $\gamma$ indicated with $\bullet, \mu$ with + , and $\nu$ with $*$.

If $\alpha=2^{4}$ and $\beta=4^{3}$, then let $\lambda=(6,5,5,2,1,1,0)$ with $\mu=(2,1,1)$ and $\nu=(1,1,0)$. See Figure 6.

If $\alpha=4^{4}$ and $\beta=3^{2}$, then let $\lambda=(6,4,4,4,3,1)$ with $\mu=(2,0)$ and $\nu=(3,1)$. See Figure 7.

The following theorem, which appears in the work of Kostant and of Littelmann, easily follows from the Littlewood-Richardson rule.

Theorem 50. Let $\alpha$ and $\beta$ be a pair of rectangles. Then $c_{\alpha, \beta}^{\lambda}=1$ if $\lambda$ is $(\alpha, \beta)$ semi-selfcomplementary and is 0 otherwise. 


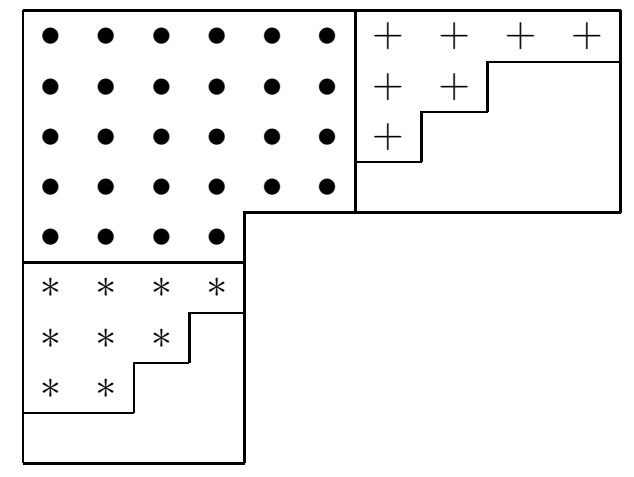

Figure 5: A $\left(6^{4}, 4^{5}\right)$ semi-self-complementary shape

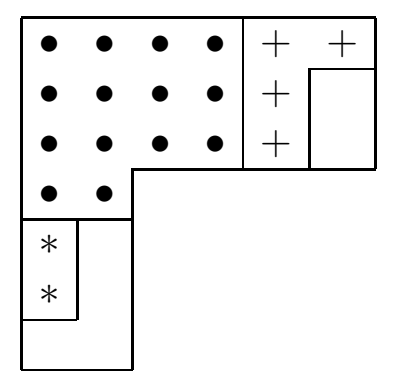

Figure 6: A $\left(2^{4}, 4^{3}\right)$ semi-self-complementary shape

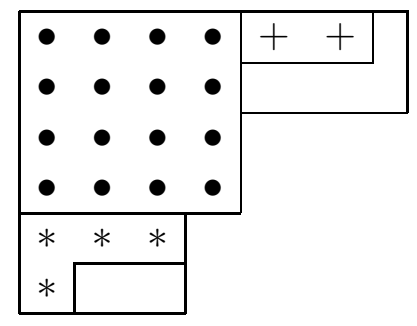

Figure 7: A $\left(4^{2}, 3^{2}\right)$ semi-self-complementary shape 
Next we define the statistic twist on $(\alpha, \beta)$ semi-self-complementary shapes. Suppose $\lambda$ is a semi-self-complementary shape with associated $\mu$ and $\nu$. Then

$$
t w(\lambda)=|\nu|
$$

In the previous examples, $t w(10,8,7,6,4,4,3,2,0)=9, t w(6,5,5,2,1,1,0)=2$ and $t w(6,4,4,4,3,1)=4$.

Note that there is exactly one $(\alpha, \beta)$ semi-self-complementary shape with twist 0 . This shape is

$$
\alpha+\beta=\left(\alpha_{1}+\beta_{1}, \alpha_{2}+\beta_{2}, \ldots\right) .
$$

\section{2 $q$-Littlewood-Richardson Coefficients for Pairs of Rectangles}

Suppose $D$ is a fixed domino tableau of $(\alpha, \beta)$ d-rectangular shape. The domino tableau $D$ will play the role of $R$ in the construction of Subsection 5.2. According to Theorem 45 and Theorem 50 there is a bijection between domino tableaux $T$ of $(\alpha, \beta)$ d-rectangular shape and standard tableaux $Q_{T}$ of the different $(\alpha, \beta)$ semi-self-complementary shapes. Furthermore, the spin of $T$ corresponds in a natural way to the number of minus signs in $P_{T}$.

Also according to Theorem 50 there will be a one-to-one correspondence between the $(\alpha, \beta)$ semi-self-complementary shapes and standard tableaux $P_{T}$. Each $P_{T}$ will have a different semi-self-complementary shape. In this subsection we will show that the number of minus signs in $P_{T}$ (and hence the spin of $T$ ) will correspond in a predictable way to the twist of the semi-self-complementary shape.

We therefore seek to identify a particular $D$ and the corresponding set of $P_{T}$. Since there is one $P_{T}$ for each semi-self-complementary shape, we will write $P[\lambda]$ for this $P_{T}$, where $\lambda$ is semi-self-complementary.

Our proof will proceed as follows. We will produce a domino tableau $D$ which has d-rectangular shape. We will then construct $P_{D}$. That is, we will find the semistandard tableau $P_{D}$ such that $P_{D}=P_{s}\left(\mathbf{w}_{D}^{\text {neg }}\right)$ where $P_{r}\left(\mathbf{w}_{D}\right)=D$ and $Q_{r}\left(\mathbf{w}_{D}\right)=D$. We will show that the shape of $P_{D}$ is $\alpha+\beta$, and so we will define $P[\alpha+\beta]=P_{D}$.

We will then prove that $P[\alpha+\beta]$ is endowed with a particular set of properties. These properties will enable us to describe the $P[\lambda]$ as a certain construction applied to $P[\alpha+\beta]$. We will then show that all the $P[\lambda]$ so constructed will be all possible $P_{T}$.

Since $D$ is domino, $P_{r}\left(\mathbf{w}_{D}\right)=P_{d}\left(\mathbf{w}_{D}\right)$ and $Q_{r}\left(\mathbf{w}_{D}\right)=Q_{d}\left(\mathbf{w}_{D}\right)$. We therefore will view $P_{r}\left(\mathbf{w}_{D}\right)$ as domino insertion, as described in Subsection 4.3 , and $Q_{r}\left(\mathbf{w}_{D}\right)$ as the corresponding domino recording tableau.

To construct $D$, we first construct two semistandard tableaux, $U$ and $V$, of shapes $\alpha$ and $\beta$ respectively. We then construct the ribbon tableau $R=U * V$. The tableau $D$ will be the standardization of $R$.

Suppose $\alpha=l^{k}$ and $\beta=n^{m}$ and suppose $k \geq m$ (the case where $m>k$ is treated in a similar manner). Let $U$ and $V$ be the unique pair of tableaux of shapes $\alpha$ and $\beta$ such 
that the total content of $U$ and $V$ is

$$
(\overbrace{l, l, \ldots, l}^{k-m}, \overbrace{l+n, l+n, \ldots, l+n}^{m}) \text {. }
$$

We now use the definition of $U * V$ to make some observations about $R$. We call the shape $(j, j-1, \ldots, 1)$ a $j$-triangle. We will assume $n \geq l$. The case when $n<l$ is somewhat simpler.

Lemma 51. The entries $1,2, \ldots, k-m$ in $R$ form a subtableau $A$ whose shape is a $(k-m) \times(2 l-k+m)$ rectangle with $a(k-m-1)$-triangle to its right and $a(k-m)$ triangle below. The entries $i, i=k-m+1, \ldots, k$, form ribbons $R_{1}, R_{2}, \ldots, R_{m}$. The ribbon $R_{1}$ has height 2 in each column from the first column to column $2 l$, and height 1 thereafter. Similarly, the ribbon $R_{2}$ has height 2 from the first column to column $2 l+1$, and height 1 thereafter, etc.

We illustrate Lemma 51 with an example. Suppose $k=5, l=3, m=3$ and $n=6$. First,

$$
U=\begin{array}{lll}
1 & 1 & 1 \\
2 & 2 & 2 \\
3 & 3 & 3 \\
4 & 4 & 4 \\
5 & 5 & 5
\end{array}
$$

and

$$
V=\begin{array}{llllll}
3 & 3 & 3 & 3 & 3 & 3 \\
4 & 4 & 4 & 4 & 4 & 4 \\
5 & 5 & 5 & 5 & 5 & 5
\end{array}
$$

Then $R$ looks like this.

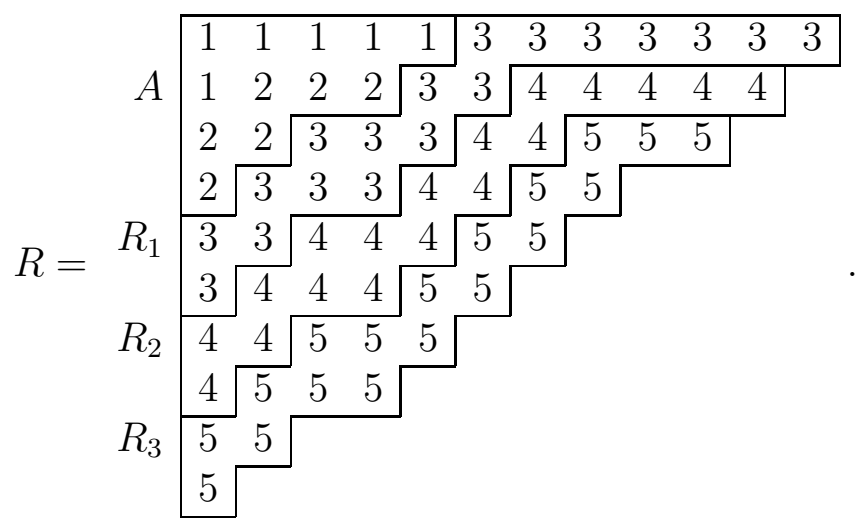

Note that when we standardize, each ribbon $R_{i}$ contains a contiguous strip of successively larger values, and the leftmost $2 l+i-1$ columns of each ribbon $R_{i}$ consists of all vertical dominoes. 
In our example,

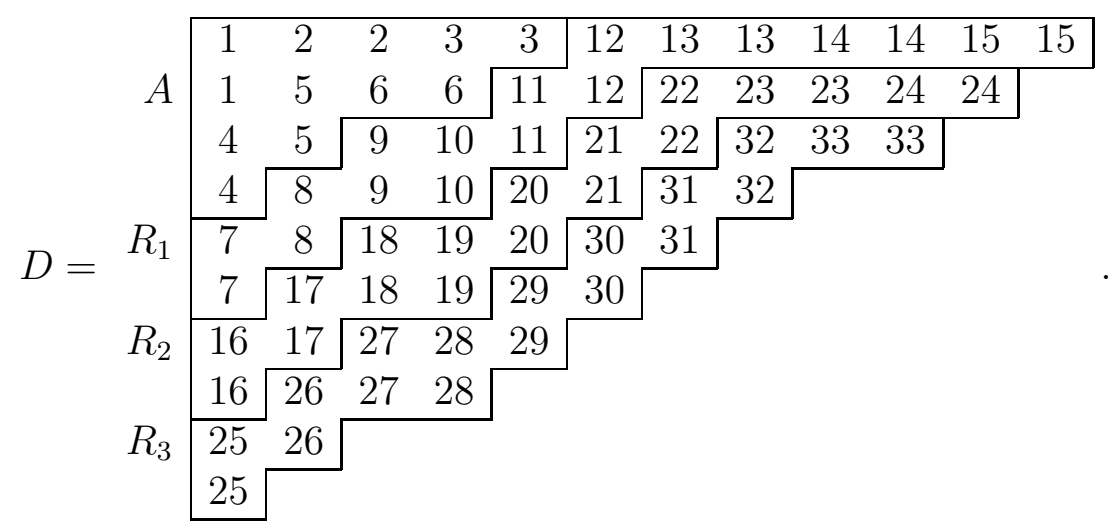

Now we consider what happens when we form $\mathbf{w}=\mathbf{w}_{D}$ such that $D=P_{r}(\mathbf{w})$ and $D=Q_{r}(\mathbf{w})$. Consider the diagonal whose intersection with the first row of $D$ is the cell just after the first row of $A$. Strictly above this diagonal all dominoes are horizontal while weakly below it, all dominoes not in $A$ are vertical. The horizontal dominoes all get bumped out to the north under reverse domino insertion. They form a triangle of dominoes which we will call $C^{+}$.

It is now easy to see from the definition of domino insertion, from our construction of $D$ and from Lemma 51 that all of the entries in the ribbons $R_{i}$ will be removed from $D$ before any entry in $A$. The entries in $C^{+}$will be bumped out horizontally, while the other entries in the ribbons $R_{i}$ will be bumped out vertically.

Let $\mathbf{w}_{R}$ denote the colored permutation resulting from the removal of these ribbons, and let $\mathbf{w}_{A}$ denote the colored permutation resulting from the removal of $A$. Thus, we write $\mathbf{w}=\mathbf{w}_{A} \mathbf{w}_{R}$. In our example,

$$
\mathbf{w}_{A}=\left(\begin{array}{llllll}
\overline{1} & 2 & 6 & \overline{5} & \overline{4} & 3
\end{array}\right)
$$

and

$$
\mathbf{w}_{R}=\left(\begin{array}{llllllllllllllllll}
\overline{12} & \overline{11} & \overline{10} & \overline{9} & \overline{8} & \overline{7} & 13 & 23 & 33 \\
& & & \overline{22} & \overline{21} & \overline{20} & \overline{19} & \overline{18} & \overline{17} & \overline{16} & 14 & 24 & & & & \\
& & & & & & & \overline{32} & \overline{31} & \overline{30} & \overline{29} & \overline{28} & \overline{27} & \overline{26} & \overline{25} & 15
\end{array}\right) .
$$

In other words, $R=\left(A \stackrel{\mathrm{d}}{\leftarrow} \mathbf{w}_{R}\right)$ and $A=P_{d}\left(\mathbf{w}_{A}\right)$. Next, let

$$
B=P_{s}\left(\mathbf{w}_{A}^{\mathrm{neg}}\right)
$$

and

$$
C=P_{s}\left(\mathbf{w}_{R}^{\text {neg }}\right) .
$$

In our example,

$$
B=\begin{array}{ccc|}
-5 & -4 & 3 \\
-1 & 2 & 6 \\
\hline
\end{array}
$$


and

$$
C=\begin{array}{ccccccccc|}
-32 & -31 & -30 & -29 & -28 & -27 & -26 & -25 & 15 \\
-22 & -21 & -20 & -19 & -18 & -17 & -16 & 14 & 24 \\
-12 & -11 & -10 & -9 & -8 & -7 & 13 & 23 & 33 \\
\hline
\end{array}
$$

Reading the diagonals of $C^{+}$gives the positive elements in the tableau $C$ by rows. The vertical dominoes which appear in a given $R_{i}$ then appear negated and reversed in the $(m-j)$-th row of $C$.

The tableaux $B$ and $C$ thus have the following properties.

i. The tableau $B$ is rectangular of dimension $(k-m) \times l$. This is because the 2 -quotient of $A$ is a single rectangle.

ii. The tableau $C$ is rectangular of dimension $m \times(l+n)$. For, from the above discussion, $\mathbf{w}_{R}^{\text {neg }}$ is the row-reading word of $C$.

iii. The subtableau of $C$ consisting of the first $2 l$ columns is entirely negative. This follows from the fact that each ribbon $R_{i}$ has at least $2 l$ vertical dominoes.

iv. The entries of $B$ are all greater than these first $2 l$ columns of $C$.

Therefore, the tableau $P[\alpha+\beta]=P_{s}\left(\mathbf{w}^{\text {neg }}\right)$ will consist of the rectangle $C$ with the rectangle $B$ directly below. In our example,

$$
P[\alpha+\beta]=\left[\begin{array}{ccccccccc}
-32 & -31 & -30 & -29 & -28 & -27 & -26 & -25 & 15 \\
-22 & -21 & -20 & -19 & -18 & -17 & -16 & 14 & 24 \\
-12 & -11 & -10 & -9 & -8 & -7 & 13 & 23 & 33 \\
-5 & -4 & 3 & & & & & & \\
-1 & 2 & 6 & & & & & &
\end{array} .\right.
$$

Next, let $r=\min \{k, m\}$ and let $s=\min \{l, n\}$ and let $\tau=\alpha \cap \beta$. Thus, $\tau=s^{r}$. Let $S$ be the part of $P[\alpha+\beta]$ in $\tau$. Let $E$ be the portion of $P[\alpha+\beta]$ to the right of $S$, that is, the first $r$ rows of $P[\alpha+\beta]$ beyond column $s$. Let $L$ be the portion of $P[\alpha+\beta]$ below row $r$. Here is a picture of these definitions.

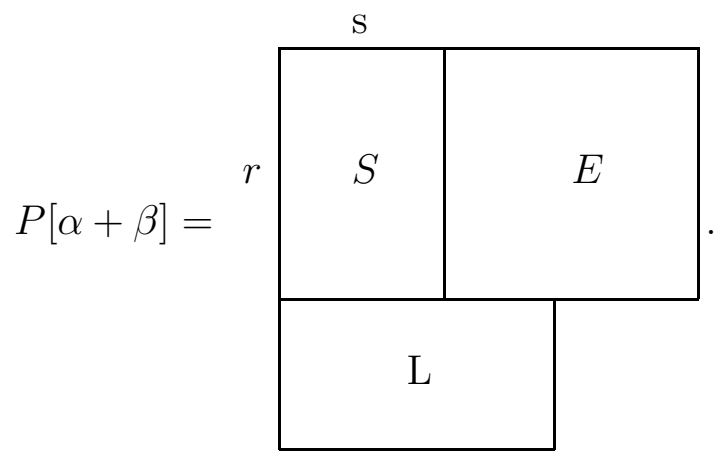

Here is the list of properties subscribed to by $P[\alpha+\beta]$. 


\section{Lemma 52.}

i. All the entries in $S$ are negative.

ii. The tableaux $E$ and $L$ are rectangular.

iii. If the tableau $L$ is placed below the tableau $E$, the resulting tableau is standard.

$i v$. If the tableau $S$ is rotated $180^{\circ}$, negated, and placed below $L$, the resulting tableau is standard.

Proof. This lemma follows from the above discussion about the construction of $P[\alpha+$ $\beta]$.

Now we will describe how all of the $P[\lambda]$ are constructed. Let $\mu$ and $\nu$ be as described in the definition of the semi-self-complementary shape $\lambda$. A general $P[\lambda]$ will have the following form:

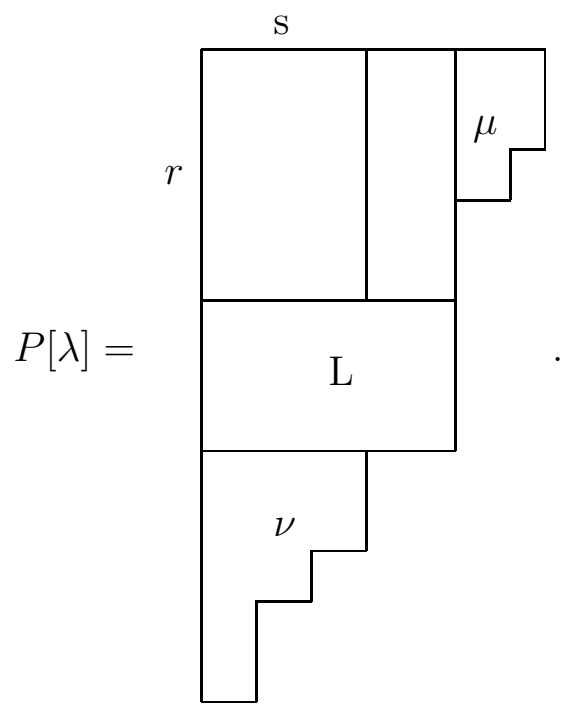

Let $P=P[\lambda]$ and pick any "corner" cell in the shape $\mu$ in $P$, say $\left(i_{c}, j_{c}\right)$.

Algorithm 2.

find $x$ and $A$ such that

$$
\begin{aligned}
& (x \stackrel{\mathrm{s}}{\rightarrow} A)=P \\
& \operatorname{sh}(P) / \operatorname{sh}(A)=\left(i_{c}, j_{c}\right) \\
& i_{x}:=\operatorname{row} \text { of } x \text { in } P \\
& \operatorname{cyc}(P):=(-x \stackrel{\mathrm{s}}{\rightarrow} A) \\
& i_{-x}:=\text { row of }-x \text { in } \operatorname{cyc}(P) \\
& \left(i_{-c}, j_{-c}\right):=\operatorname{sh}(\operatorname{cyc}(P)) / \operatorname{sh}(A)
\end{aligned}
$$


We illustrate Algorithm 2 here. Let

$$
P[\lambda]=\begin{array}{ccc|cc|}
-17 & -16 & -15 & -14 & -13 \\
-11 & -10 & -9 & -8 & -7 \\
-4 & -3 & -2 & -1 & \\
\hline 5 & 6 & & \\
12 & & & \\
18 & &
\end{array} .
$$

Let $i_{c}=3$ and $j_{c}=4$. Then $x=-4$ and

$$
A=\begin{array}{ccccc}
-17 & -16 & -15 & -14 & -13 \\
-11 & -10 & -9 & -8 & -7 \\
-3 & -2 & -1 & & \\
5 & 6 & & & \\
12 & & & & \\
18 & & & &
\end{array} .
$$

Inserting 4 into $A$ gives

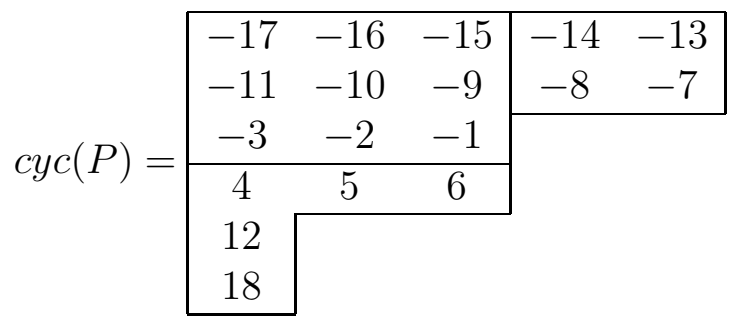

This algorithm has a natural "inverse": Find a corner cell in the shape $\nu$ and use it to begin reverse column insertion. Remove $x$, negate it, and reinsert it.

We now make the following observations about Algorithm 2, based on Lemma 52. As noted earlier, the operator neg on colored words is invertible. Let bar denote the inverse of this operator.

\section{Lemma 53.}

i. The entry $x$ is negative (positive in the inverse algorithm).

ii. The rows $i_{x}$ and $i_{c}$ are equal.

iii. The rows $i_{-x}$ and $i_{-c}$ are equal.

iv. We have $i_{x}+i_{-x}=m+k+1$.

$v$. The tableau $\operatorname{cyc}(P)=P[\operatorname{cyc}(\lambda)]$ where $\operatorname{cyc}(\lambda)$ is obtained from $\lambda$ by removing a single corner cell in $\mu$ and placing it in the complementary position in $\nu$.

vi. Let $\mathbf{w}$ be a word such that $P=P_{s}(\mathbf{w})$ and let $\mathbf{v}$ be a word such that $\operatorname{cyc}(P)=P_{s}(\mathbf{v})$. Then $\mathbf{w}^{\mathrm{bar}} \stackrel{\mathrm{r}}{\sim} \mathbf{v}^{\text {bar }}$. 
Proof. Items (i)-(v) follow from Lemma 52. For item (vi), recall that $P=(x \stackrel{\mathrm{s}}{\rightarrow} A)$ and $\operatorname{cyc}(P)=(-x \stackrel{\mathrm{s}}{\rightarrow} A)$. Now do a reverse column insertion from row $i_{-x}-1$ of $A$. This is possible since $A$ can be constructed by a reverse column insertion from row $i_{-x}$. Let $y$ be the letter thus removed and $B$ the resulting tableau. By Lemma 52, we have $y>0$ and $y<-x$. Also, since $A=(y \stackrel{\mathrm{s}}{\rightarrow} B)$, we have $P=(x \stackrel{\mathrm{s}}{\rightarrow}(y \stackrel{\mathrm{s}}{\rightarrow} B))$ and $\operatorname{cyc}(P)=(-x \stackrel{\mathrm{s}}{\rightarrow}(y \stackrel{\mathrm{s}}{\rightarrow} B))$.

Therefore, by Corollary $5, w(P) \stackrel{\mathrm{s}}{\sim} x y w(B)$ while $w(\operatorname{cyc}(P)) \stackrel{\mathrm{s}}{\sim}-x y w(B)$. By Corol-

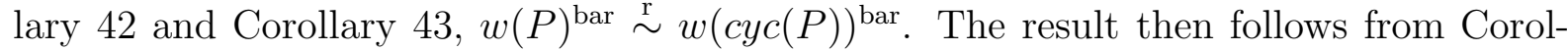
lary 42 and Corollary 5.

Our construction has produced an injection from $(\alpha, \beta)$ semi-self-complementary standard tableaux to domino tableaux of $(\alpha, \beta)$ d-rectangular shape. That this is, in fact, a bijection follows now from Theorem 50, which shows that these two sets are bijectively equivalent.

Furthermore, $P[\operatorname{cyc}(\lambda)]$ has one less minus sign than $P[\lambda]$. By Theorem 45, sp(T) must decrease by one. On the other hand, the above discussion shows that in going from $\lambda$ to $\operatorname{cyc}(\lambda)$, twist increases by one. But the minimum twist and the minimum cospin are both 0 . Thus, $\operatorname{cosp}(T)=t w(\lambda)$.

Note that since one domino recording tableau corresponding to $P[\alpha+\beta]$ is $D$, we must also have $\operatorname{cosp}(D)=0$.

Theorem 54. The bijection from ribbon tableau $T$ of $(\alpha, \beta)$-rectangular shape to $(\alpha, \beta)$ semi-self-complementary semistandard tableau $Q_{T}$ of shape $\lambda$, defined by $Q_{T}=T^{\mathrm{ev}}$, satisfies

$$
\operatorname{cosp}(T)=t w(\lambda)
$$

We illustrate Theorem 54 as follows. Let $\alpha=4^{2}$ and $\beta=3^{3}$ and let

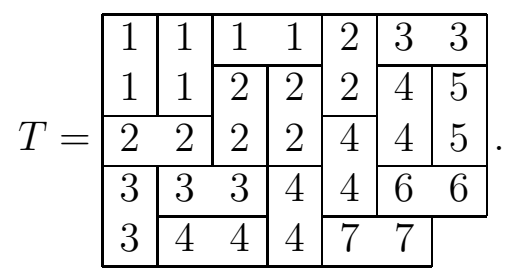

We have $\operatorname{cosp}(T)=2$. Then

$$
Q_{T}=T^{\mathrm{ev}}=\begin{array}{lllll|lll|}
1 & 1 & 1 & 2 & 2 & 3 & 5 \\
2 & 2 & 4 & 4 & 4 & & \\
3 & 3 & 6 & & & & \\
\cline { 1 - 2 } 4 & 7 & & & & & \\
\hline & & & & & &
\end{array}
$$

so that $\lambda=(7,5,3,2,0)$ and $t w(\lambda)=2$. 


\section{Remarks}

The domino insertion algorithm described in Subsection 4.3 shares many features with the more general ribbon insertion algorithm of [22]. Both insertions can be placed into a Fomin-like array-of-tableaux context.

While the new Littlewood-Richardson bijection given in Subsection 5.2 is quite easily described, and the spin of the tableau is the same for the entire recording equivalence class, it does happen that the same shape occurs more than once from ribbon tableaux with different spins. The rectangular case happened to have multiplicity-free LittlewoodRichardson coefficients, and the spin statistic translated to a simple statistic on the shape. This is not always the case. For instance, if $\mu=\nu=(2,1)$ and $\lambda=(3,2,1)$, then $c_{\mu, \nu}^{\lambda}(q)=q+q^{2}$. Thus each standard tableau of shape $(3,2,1)$ occurs twice as a $Q_{T}$, and the coimage of such a tableau consists of two ribbon tableaux with differing spins. To identify this spin requires knowledge of the tableaux $P_{T}$.

The Littlewood-Richardson bijection in Subsection 5.2 is somewhat unusual in that it holds the insertion tableau fixed and lets the recording tableau range over all ribbon tableaux. In proofs of the Littlewood-Richardson rule, the opposite is usually done. A dual version of Theorem 45 should be possible, wherein the recording tableau is held constant and the insertion tableau varies. This dual version will identify the insertion tableau with a pair of semistandard tableaux, but this identification will not be directly through the usual RSK correspondence. We leave for the reader to work out the details of this dual version.

It would be interesting to find an algorithm for generating the list of $P_{T}$. Such an algorithm would be a new Littlewood-Richardson rule which would keep track of the power of $q$ in $c_{\mu, \nu}^{\lambda}(q)$. One method, by no means complete, is the "cyclage" method described in Subsection 6.2.

It should be possible to generalize many of the results in this paper to shapes with non-empty 2-core, as in [27].

Corollaries 42 and 43 were needed in the proof of Theorem 54. These corollaries are not sufficient to define ribbon equivalence completely. For instance, $\overline{1} 4 \overline{2} 3 \stackrel{\mathrm{r}}{\sim} \overline{1} 43 \overline{2}$, as can be easily seen by applying Theorem 41 or by simply applying the domino insertion of Subsection 4.3 directly. However, this equivalence cannot be obtained by a sequence of switches as described in Corollaries 42 and 43.

\section{Acknowledgments}

The authors wish to thank the referee for many useful comments, suggestions and recommendations. The second author benefitted greatly from several helpful conservations with Vic Reiner. 


\section{References}

[1] D. Barbasch and D. Vogan, Primitive ideals and orbital integrals on complex classical groups, Math. Ann. 259 (1982), pp. 153-199

[2] A. Berenstein and A. Kirillov, Schützenberger involution and action of the symmetric group Proceedings of 10th International Conference on Formal Power Series and Algebraic Combinatorics, Fields Institute, Toronto, 1998

[3] C. Carré and B. Leclerc, Splitting the square of a Schur function into its symmetric and antisymmetric parts, J. Alg. Combin. 4 (1995), pp. 201-231.

[4] S. Fomin, Schensted algorithms for dual graded graphs, J. Algebraic Combin. 4 (1995), pp. 5-45.

[5] S. Fomin and D. Stanton, Rim hook lattices, St. Petersburg Math. J. 9 (1998), pp. 1007-1016.

[6] W. Fulton, Young Tableaux, London Mathematical Society, 1997.

[7] D. Garfinkle, On the classification of primitive ideals for complex classical Lie algebras, I, Compositio Mathematica 75 (1990), pp. 135-169.

[8] M. D. Haiman, On mixed insertion, symmetry, and shifted Young tableaux, J. Combin. Theory Ser. A 50 (1989), pp. 196-225.

[9] G. James and A. Kerber, Representation Theory of the Symmetric Group, Addison Wesley, 1981.

[10] D. Knuth, Permutations, matrices, and generalized Young tableaux, Pacific J. of Math. 34 (1970), pp. 709-727.

[11] A. Lascoux, B. Leclerc and J.-Y. Thibon, Ribbon tableaux, Hall-Littlewood functions and unipotent varieties, Sém. Lothar. Combin., 34 (1995).

[12] A. Lascoux and M. P. Schützenberger, Le monö̈de plaxique, Non-Commutative Structures in Algebra and Geometric Combinatorics, Quaderni de "La ricerca scientifica," n. 109, Roma, CNR, (1981), pp. 129-156.

[13] D. E. Littlewood, Modular representations of symmetric groups, Proc. Royal Soc. London Ser. A, 209 (1951), pp. 333-353.

[14] I. Macdonald, Symmetric Functions and Hall Polynomials, Oxford University Press, 1995.

[15] T. Nakayama, On some modular properties of irreducible representations of a symmetric group, I, Jap. J. Math. 18 (1941), pp. 89-108. 
[16] T. Nakayama, On some modular properties of irreducible representations of a symmetric group, II, Jap. J. Math. 17 (1941), pp. 411-423.

[17] G. de B. Robinson, On the representations of the symmetric group, Amer. J. Math. 60 (1938), pp. 745-760.

[18] G. de B. Robinson, On the representations of the symmetric group. III, Amer. J. Math. 70 (1948), pp. 277-294.

[19] B. Sagan, The Symmetric Group, Wadsworth \& Brooks/Cole, 1991.

[20] C. Schensted, Longest increasing and decreasing subsequences, Canadian J. of Math. 13 (1961), pp. 179-191.

[21] A. Schilling, M. Shimozono and D. White, Branching formula for $q$-LittlewoodRichardson coefficients, preprint,

[22] M. Shimozono and D. White, Color-to-spin ribbon Schensted algorithms, Discrete Math., to appear.

[23] M. P. Schützenberger, Quelques remarques sur une construction de Schensted, Math. Scandinavica 12 (1963), pp. 117-128.

[24] R. Stanley, Enumerative Combinatorics, Vol 2, Cambridge, 1999.

[25] R. Stanley, Symmetries of plane partitions, J. Combin. Theory Ser. A 43 (1986), pp. $103-113$.

[26] D. Stanton and D. White, A Schensted algorithm for rim-hook tableaux, J. Combin. Theory Ser. A 40 (1985), pp. 211-247.

[27] M. A. A. van Leeuwen, The Robinson-Schensted and Schützenberger algorithms, an elementary approach, Electronic J. of Combin. 3 (1996).

[28] M. A. A. van Leeuwen, Several bijective correspondences involving domino tableaux, preprint.

[29] D. White, Sign-balanced posets, J. Combin. Theory, Ser. A, to appear.

[30] D. White, Some connections between the Littlewood-Richardson rule and the construction of Schensted, J. Combin. Theory, Ser. A 30 (1981), pp. 237-247. 